\title{
Quantified Epistemic Logics for Reasoning about Knowledge in Multi-Agent Systems
}

\author{
F. Belardinelli \\ Scuola Normale Superiore \\ Pisa, Italy \\ A. Lomuscio \\ Department of Computing \\ Imperial College London, UK
}

\begin{abstract}
We introduce quantified interpreted systems, a semantics to reason about knowledge in multi-agent systems in a first-order setting. Quantified interpreted systems may be used to interpret a variety of first-order modal epistemic languages with global and local terms, quantifiers, and individual and distributed knowledge operators for the agents in the system. We define first-order modal axiomatisations for different settings, and show that they are sound and complete with respect to the corresponding semantical classes.

The expressibility potential of the formalism is explored by analysing two MAS scenarios: an infinite version of the muddy children problem, a typical epistemic puzzle, and a version of the battlefield game. Furthermore, we apply the theoretical results here presented to the analysis of message passing systems $[17,41]$, and compare the results obtained to their propositional counterparts. By doing so we find that key known meta-theorems of the propositional case can be expressed as validities on the corresponding class of quantified interpreted systems.
\end{abstract}

\section{Introduction}

Modal logics for reasoning about knowledge, or modal epistemic logics, have been widely studied in the artificial intellegence community for several years.

Email addresses: f.belardinelli@sns.it (F. Belardinelli), A.Lomuscio@imperial.ac.uk (A. Lomuscio). 
The interest in this area is directed both towards fundamental theoretical research, covering topics such as axiomatisability, completeness, decidability, and complexity of these formalisms [6-8], as well as to the application of modal epistemic languages to the specification and verification of multi-agent systems $[23,55,66]$.

The typical epistemic language extends propositional logic by adding $n$ modalities $K_{i}$ representing the knowledge of agent $i$ in a group $A=\{1, \ldots, n\}$ of agents. The use of these formalisms as specification languages is nowadays so mainstream as to require little justification: these are rather expressive languages, well-understood from a theoretical point of view $[17,51]$.

For expressiveness purposes epistemic logic has been extended in several directions. In one line of research modalities for distributed and common knowledge have been added to the basic formalism $[17,18]$. In another one, the epistemic language has been enriched with temporal operators under the assumption of a given model of time, e.g., linear or branching, discrete or continuous $[32,50,72]$. In yet another direction, epistemic logic has been studied at the predicate level $[39,46,53]$, thus allowing for richer specifications.

This paper takes inspiration from this last line of research, and is intended to contribute to the subject of first-order epistemic logic for the specification of multi-agent systems (MAS). Specifically, we provide first-order epistemic languages with a computationally grounded semantics [67] given in terms of quantified interpreted systems (QIS). We believe that QIS are a natural extension to first-order of interpreted systems, the typical semantical framework for epistemic logics in MAS $[17,55]$. Further, we analyse from an epistemic perspective the theoretical implications of different first-order extensions: quantification domains indexed to agents and to computational states, domains of intensional obejcts, etc. One of the main results of this paper consists in showing that all the semantical classes introduced are finitely axiomatisable. This is obtained by adapting standard techniques in quantified modal logic $[19,22,33]$ to the epistemic frameworks here analysed. We also show that the techniques in [18] for proving completeness of epistemic languages with distributed knowledge can be extended to first-order. Finally, the formalisms presented allow to study in a first-order setting message passing systems, a typical framework in distributed systems $[41,54]$, revealing that meta-theorems on message passing systems can be expressed as validities in the corresponding class of quantified interpreted systems.

\subsection{First-order Epistemic Logic in Knowledge Representation}

Although quantified modal logic (QML) has a reputation for being a source of incompleteness [22,59], quantification is an expressive feature that has often been considered in the literature on knowledge representation and AI. For instance, when specifying the behaviour of MAS, quantification is so natural 
and convenient that one feels compelled to bring it explicitly into the syntax. As it is maintained in [42]:

"the power of FOL [first-order logic] is used not so much to deal with infinities but to deal with incomplete knowledge." ([42], p. 81)

Moreover, in knowledge representation the power of first-order logic is welcome every time agents reason about:

- relational statements, as in "agent $i$ knows that message $\mu$ was sent by agent $a$ to agent $b "$, or formally,

$$
K_{i} \operatorname{Sent}(a, b, \mu)
$$

- functional dependencies and identity, as in "agent $i$ knows that message $\mu$ is the encryption of message $\mu^{\prime}$ with key $k$ ", or formally,

$$
K_{i}\left(\mu=\operatorname{enc}\left(k, \mu^{\prime}\right)\right)
$$

- infinite domains of individuals, or finite domains whose cardinality cannot be bounded in advance: "agent $i$ has to read any e-mail before deleting it",

$$
\forall \mu(\operatorname{Delete}(i, \mu) \rightarrow \operatorname{Read}(i, \mu))
$$

- quantification on agents: "the child of any process knows who launched it"

$$
\forall i K_{\text {child }(i)} \text { Launched }(i, \operatorname{child}(i))
$$

Furthermore, in the context of logics for knowledge it is well known that epistemic modalities can be combined with quantifiers to formalise de re/de dicto knowledge [19,33], expressing concepts not expressible at the propositional level. For instance, agent $i$ might know that every computation produces an output, thus having the de dicto knowledge expressed in the following specification:

$$
\forall \text { comp } K_{i} \exists y \text { Output(comp,y) }
$$

but he might not know the actual output of every computation. Therefore, the following de re specification would not be satisfied:

$$
\forall c o m p \exists y K_{i} \text { Output(comp,y) }
$$

However, the use of first-order modal logic in MAS specifications is sometimes frowned upon: why should we use an undecidable language when a decidable one does the job reasonably well already? Is the price that quantification brings in justified? While these objections are certainly sensible, we believe that their strength has been increasingly weakened by recent progress in MAS verification by model checking $[21,56]$ and advances in the theory of QML. In the model checking approach $[9,34,49]$ the decision problem is tackled not 
by checking validity, but simply by considering model satisfaction. In other words, we do not check whether a formula representing a specification is satisfiable in some model, but whether it is true on the model representing all possible evolutions of a given system. While the former problem is undecidable for QML, the latter might be decidable at least for many interesting fragments, further investigations being required.

In addition, recent contributions on axiomatisability $[60,65]$ and decidability $[28,30,64]$ of quantified modal logic, including both positive $[14,26,27,58]$ and negative results $[15,29,61,63]$ have shown that useful QML fragments are axiomatisable and even decidable, raising hopes that some quantified MAS logic may also be axiomatisable and decidable.

Our motivation for the present contribution comes from an interest in reasoning about multi-agent systems, whose high-level properties may usefully be modeled by epistemic formalisms suitably extended to predicate logic. Before turning to the formal machinery, we review the main approaches to first-order epistemic formalisms in knowledge representation and artificial intelligence.

\subsection{State of the Art}

The study of epistemic logic in a first-order setting has a long and established tradition in AI. Among the early contributions we highlight [20,25], where some semantical and philosophical issues about identity in epistemic contexts are explored. More recent works focus on the computational and planning abilities of agents $[42,52]$, where first-order epistemic logic (FOEL) is used as a specification language for agents. In [53] a theory of action is presented, which takes into account the knowledge prerequisites to action and the effects of action on knowledge.

First-order logic has also been extensively applied to the deductive machinery of knowledge bases. In [39,40] a logic of belief on a decidable form of firstorder entailment is presented, and in $[44,45]$ these investigations are further pursued by considering restricted forms of first-order doxastic logic. FOEL is also briefly discussed in classic textbooks [17,51], along with its Kripke semantics and some significant validities, even though there is no attempt to extend interpreted systems to first-order, nor completeness issues are tackled. Also, $[35,36]$ introduces applications of FOEL to game theory, while $[5,10]$ discusses epistemic formalisms in the context of security protocol. In an orthogonal dimension to the above, [24] presents an analysis of common knowledge in multi-agent systems with infinitely many agents.

First-order epistemic logics for MAS specification are used in [69-71], where they are studied mostly in relation to the MAPLE programming language for agents. In [69] the quantified epistemic temporal logic QUETL is presented in order to develop a verification methodology for multi-agent systems. Firstorder logic appears as the base of a number of other agent theories, such as 
$B D I$ logics [57], the $K Q M L$ framework [11], and the $\mathcal{L O} \mathcal{R} \mathcal{A}$ framework [68], together with modalities for mental attitudes (knowledge, belief, intention, desire), as well as temporal and dynamic operators. However, most of the current literature on agent theories have so far fallen short of a systematic analysis of the formal machinery underlying these frameworks. Often the theoretical properties (axiomatisability, decidability, complexity) of these MAS formalisms are not the main focus of these investigations. Indeed, certain frameworks are so rich that it is extremely unlikely to devise any finite axiomatisation. Still, these references are relevant for the present investigations as they are among the few to have explicitly addressed the subject of first-order modal languages in a MAS setting.

From a purely theoretical perspective, quantified modal logic has received much attention with a wealth of contributions on axiomatisability $[14,60,65]$, decidability $[15,28,64]$, and complexity $[27,30]$ of fragments of QML. In [64] the monodic fragment of quantified modal logic, where at most one free variable appears in the scope of modal operators, is introduced, and decidability of various subfragments is proved. In $[28,26]$ similar results are obtained for monodic fragments of first-order temporal logic, while the complexity of some of these formalisms is analysed in [27,30]. Further, [65] provides a complete axiomatisation of monodic first-order validities on the natural numbers. Also the monodic fragment of FOEL has been explored. In [63] monodic fist-order epistemic logic with common knowledge is proved to be decidable, and in $[60,61]$ this fragment is axiomatised.

The lines of work above constitute the theoretical and applied background against which this research is set.

\subsection{The Present Contribution}

This paper extends the current state of the art in first-order epistemic logic by introducing a family of provably complete first-order epistemic logics with global and local terms. While the denotation of the former is rigid, i.e., it is the same in every computational state, the latter's depends on the state in which these expressions are interpreted. The quantified epistemic languages here introduced are non-trivial extensions of the propositional modal languages in $[19,33]$. The use of flexible terms is inspired by [22], which also presents a completeness proof for such languages. The relevance of the distinction between global and local terms has since long been recognized, see, e.g., [19,48]. From a semantical viewpoint the key contribution of the paper is the extension of interpreted systems to first-order, obtained by endowing these structures with quantification domains. In quantified interpreted systems we allow each agent to reason about a possibly different set of objects. This choice is motivated by the fact that, as agents are autonomous, they may be aware of only a subset of all existing individuals, possibly different from those of other agents. 
We do not aim to provide a theory of awareness about agents' knowledge, but simply observe that most universally quantified statements refering to an agent $i$, such as "all boxes are red", are really universal only if $i$ is assumed to consider a proper subset of the domain of all possible individuals: the set of boxes considered by $i$. Interpreted systems are the typical formalism for epistemic logic in MAS [23,55], but to our knowledge there has been no attempt to extend these structures to first-order, even in references such as [17] where FOEL and interpreted systems are explicitly considered. We deem this extension potentially of note as these structures represent a computationally grounded semantics in the sense of [67], suitable for the definition of protocols and programs for agents, and for the development of model checking techniques.

Furthermore, provably sound and complete axiomatisations are presented for all classes of QIS here considered. The completeness proofs make use of standard techniques in QML $[12,62]$ suitably extended to deal with the semantical framework of quantified interpreted systems and a richer language with local terms, indexed quantifiers, and distributed knowledge. The completess proof for FOEL with distributed knowledge extends to first-order the techniques in [18]. Note that an indipendent completeness proof for these propositional languages appeared in [31].

Finally, we apply the formal machinery to the analysis of MAS scenarios. The muddy children puzzle and message passing systems belong to the folklore of AI and distributed systems $[17,41]$, but their first-order formalisations here provided are original.

Scheme of the paper. In Section 2 we present two classes of first-order structures: systems of global states and Kripke frames, as well as several subclasses of these. In Section 3 we introduce a group of first-order modal languages, which are interpreted on quantified interpreted systems, a valued version of systems of global states. In Section 4 we introduce a class of first-order modal axiomatisations, and prove their soundness and completeness with respect to the structures in Section 2. In Section 5 we exemplify the use of the syntax and semantics by describing some formal models for multi-agent systems and discuss some specification patterns. Section 6 is devoted to the analysis of message passing systems. Finally, Section 7 presents related work and draws some conclusions.

\section{Systems of Global States and Kripke Frames}

In this section we introduce systems of global states and Kripke frames in a first-order setting. While the first ones are used in computer science to model the behaviour of MAS $[17,23,51,55]$, Kripke frames are typically employed to 
acquire a deeper understanding of the formal properties of these systems [6-8]. Technically, we extend the corresponding propositional structures to first-order by considering a distinct domain of quantification for each agent. This choice is motivated by the fact that, as agents are autonomous, they might consider distinct domains of individuals. Then we define two maps between these classes of structures, and prove that the composition of these maps is an isomorphism. These results will be used for translating the completeness results from Kripke semantics to systems of global states. In what follows we assume a set of agents $A=\{1, \ldots, n\}$.

\subsection{Systems of Global States}

This paper is primarily concerned with the representation of knowledge in MAS, not their temporal evolution. Given this, we adopt the "static" perspective on the systems of global states [47], rather than their "dynamic" version [17]. So, while we assume that the states of the system result from the evolution given by protocols and transitions, we do not consider these explicitly. More formally, consider a set $L_{i}$ of local states $l_{i}, l_{i}^{\prime}, \ldots$, for each agent $i \in A$, and a set $L_{e}$ containing the local states of the environment $l_{e}, l_{e}^{\prime}, \ldots$ We define a system of global states as follows:

Definition 1 (Systems of Global States) A system of global states, or SGS, is a tuple $\mathcal{S}=\left\langle S, D,\left\{D_{i}\right\}_{i \in A}, F,\left\{F_{i}\right\}_{i \in A}\right\rangle$ such that $S \subseteq L_{e} \times L_{1} \times \ldots \times L_{n}$ is a non-empty set of global states; $D$ is a non-empty set of individuals, and for $i \in A, D_{i}$ is a subset of $D ; F \subseteq\{f \mid f: S \rightarrow D\}$ is a non-empty set of functions from $S$ to $D$, and for $i \in A, F_{i}$ is a subset of $F$.

$W e$ denote the class of all systems of global states as $\mathcal{S G S}$.

Intuitively, $S$ is the set of possible configurations of the system corresponding to the set of reachable states obtained by considering the temporal evolution of the system. Given that we do not consider the temporal dimension, we simply take $S$ as a subset of the set of all possible states.

The domain $D$ contains the individuals for interpreting global terms, and $F$ is the interpretation domain for local terms. We assign a fixed meaning to global terms like " $\pi$ " by including the corresponding real number in the domain $D$ of individuals. On the contrary, expressions such as "the value stored in the register", which identifies possibly different values $a$ and $a^{\prime}$ at different global states $s$ and $s^{\prime}$ respectively, will be modeled as a function $f \in F$, also called intensional object, such that $f(s)=a$ and $f\left(s^{\prime}\right)=a^{\prime}$.

In systems of global states we have possibly different quantification domains $D_{i}$ and $F_{i}$ for each agent $i \in A$. This feature is motivated by the fact that, as each agent $i$ is autonomous and may have only a partial view of the surrounding environment, she could have access only to subsets $D_{i}$ and $F_{i}$ of the domains 
$D$ and $F$ of individuals, possibly different from the domains $D_{j}$ and $F_{j}$ of another agent $j$.

Note that in general $D_{i}$ and $F_{i}$ are independent from global states, i.e., for $s, s^{\prime} \in S$, the domains $D_{i}$ and $F_{i}$ of agent $i$ in $s$ are the same as the domains $D_{i}$ and $F_{i}$ in $s^{\prime}$. This means that individuals neither appear nor disappear during the evolution of the system. We deem this assumption consistent with the external account of knowledge usually adopted in the framework of epistemic logic. In this line of research knowledge is ascribed to agents by an external observer (in our case the system specifier), rather than explicitly possessed by them. This means that a formula like $K_{i} \forall_{i} y \phi$ does not express that agent $i$ explicitly knows that all $y$ are $\phi$, i.e., that $\forall_{i} y \phi$ is the case. Instead, it suffices that for every $y, i$ knows that $\phi$ is the case, i.e., $\forall_{i} y K_{i} \phi$. In other words, agent $i$ needs not to be aware of all the individuals in his domain of quantification. In this respect the individuals in $D_{i}$ and $F_{i}$ can be thought of as persisting in the various global states from the viewpoint of the external observer.

In the next section we consider various classes of systems of global states.

\subsection{Further Systems of Global States}

We remarked that in systems of global states individuals neither appear nor disappear. A different scenario we may wish to consider consists of quantification domains changing at run-time. For this purpose we introduce varying systems of global states:

Definition 2 (Varying SGS) A varying system of global states, or varying $S G S$, is a tuple $\mathcal{S}=\left\langle S, D,\left\{D_{i}\right\}_{i \in A}, F,\left\{F_{i}\right\}_{i \in A}\right\rangle$ such that $S, D$, and $F$ are defined as in Definition 1, and for each $i \in A, D_{i}$ is a function from $S$ to the power set $\wp(D)=\left\{D^{\prime} \mid D^{\prime} \subseteq D\right\}$, and $F_{i}$ is a function from $S$ to the power set $\wp(F)=\left\{F^{\prime} \mid F^{\prime} \subseteq F\right\}$.

We denote the class of all varying systems of global states as $\mathcal{S G S}^{\text {var }}$.

In a varying SGS the domains of quantification depend not only on the agent, but also on her computational state, that is, for $s \neq s^{\prime}$, the quantification domain $D_{i}(s)$ (respectively $F_{i}(s)$ ) can possibly be different from $D_{i}\left(s^{\prime}\right)$ (respectively $F_{i}\left(s^{\prime}\right)$ ). Therefore, varying SGS are useful to model systems in which individuals might appear and disappear during the system runs.

Observe that the domains $D_{i}(s)$ and $F_{i}(s)$ do not contribute to the definition of the local state of agent $i$ in a state $s$. This implies that there can be states $s$ and $s^{\prime}$ such that $l_{i}(s)=l_{i}\left(s^{\prime}\right)$, and yet $D_{i}(s) \neq D_{i}\left(s^{\prime}\right)$ or $F_{i}(s) \neq F_{i}\left(s^{\prime}\right)$. The quantification domains of $i$ depend also on the local states of the other agents. While it is instructive to look at this general setting, it is also of interest to consider the case where the same quantification domain is available to one agent when she is in the same local state. Thus, we introduce regular systems 
of global states:

Definition 3 (Regular SGS) A regular system of global states, or regular $S G S$, is a varying $S G S$ such that:

$$
\text { for all } s, s^{\prime} \in S, l_{i}(s)=l_{i}\left(s^{\prime}\right) \text { implies } D_{i}(s)=D_{i}\left(s^{\prime}\right) \text { and } F_{i}(s)=F_{i}\left(s^{\prime}\right)
$$

We denote the class of all regular systems of global states as $\mathcal{S G S}^{\text {reg }}$.

In other words, in regular SGS the quantification domains of agent $i$ are part of her local state.

Further, note that in a system of global states the individuals in $D$ can be seen as constant functions from $S$ to $D$ itself, i.e., for every $a \in D$ consider the function $a: S \rightarrow D$ returning $a$ for every $s \in S$. Hence, we can take $D$ itself as the domain $F$ of intensional objects, thus obtaining a peculiar class of systems of global states:

Definition 4 (Objectual SGS) An objectual system of global states, or objectual $S G S$, is an $S G S \mathcal{S}=\left\langle S, D,\left\{D_{i}\right\}_{i \in A}, F,\left\{F_{i}\right\}_{i \in A}\right\rangle$ such that $D=F$, and for $i \in A, D_{i}=F_{i}$.

We denote the class of all objectual $S G S$ as $\mathcal{S G S}^{\text {obj }}$.

Objectual SGS, which can be seen as triples $\mathcal{S}=\left\langle S, D,\left\{D_{i}\right\}_{i \in A}\right\rangle$, are particular instances of SGS, easier to handle and yet suitable for modeling useful applications, as we will show in Section 5. In fact, objectual SGS are sufficient for interpreting first-order epistemic languages with global terms only, the typical formalism for quantified modal logic $[19,22,33]$. Thus, the present semantical framework encompasses many of the formal accounts available in the literature on QML.

For the present investigations it is of particular interest to explore the relationship among individuals in the various $D_{i}$ and $F_{i}$, for $i \in A$. In general system of global states the domain $F_{i}$ can be any set of function from $S$ to $D$, but we may find convenient to define some constraints on the functions in $F_{i}$. In the rest of the paper we will consider the following constraints:

for all $s, s^{\prime} \in S, l_{i}(s)=l_{i}\left(s^{\prime}\right)$ and $f(s) \in D_{i}$ implies $f\left(s^{\prime}\right) \in D_{i}$

for all $s, s^{\prime} \in S, f \in F_{i}, l_{i}(s)=l_{i}\left(s^{\prime}\right)$ and $f(s) \in D_{i}$ implies $f\left(s^{\prime}\right) \in D_{i}(3)$

for all $s \in S, \quad f \in F_{i}, f(s) \in D_{i}$

for all $s, s^{\prime} \in S, f \in F_{i}$ and $l_{i}(s)=l_{i}\left(s^{\prime}\right)$ implies $f(s)=f\left(s^{\prime}\right)$

for all $s, s^{\prime} \in S, f \in F_{i}$ and $l_{i}(s)=l_{i}\left(s^{\prime}\right)$ implies $f(s)=f\left(s^{\prime}\right) \in D_{i}$

Intuitively, formula (2) expresses the fact that if the intensional object $f$ chooses an existing individual at global state $s$, then agent $i$ knows this fact. Formula (3) restricts formula (2) to the intensional objects in the domain $F_{i}$, 
while formula (4) is a strenghtening of (3) urging that $F_{i}$ is a set of functions with domain $S$ and codomain $D_{i} \subseteq D$. Further, by formula (5) the functions in $F_{i}$ have as domain the set $L_{i}$ of agent $i$ 's local state, while formula (6) is the conjunction of (4) and (5), and it implies that the functions in $F_{i}$ have domain $L_{i}$ and codomain $D_{i}$.

Given the above, we introduce five further classes of systems of global states.

Definition 5 For every $k, 2 \leq k \leq 6, \mathcal{S G S}^{k} \subseteq \mathcal{S G S}$ is the class of the system of global states satisfying formula $(k)$.

In Section 4 we will prove that the results available for the systems of global states in $\mathcal{S G S}$, notably the soundness and completeness theorems, also apply to the other classes of SGS with minor changes. The following table summarizes the classes of SGS we will analyse in the rest of the paper, for $2 \leq k \leq 6$.

\begin{tabular}{|c|c|}
\hline \multicolumn{2}{|r|}{ Systems of Global States } \\
\hline $\mathcal{S G S}$ & all \\
\hline $\mathcal{S} \mathcal{G} \mathcal{S}^{\text {var }}$ & varying \\
\hline $\mathcal{S} \mathcal{G} \mathcal{S}^{o b j}$ & objectual \\
\hline $\mathcal{S} \mathcal{G} \mathcal{S}^{r e g}$ & regular \\
\hline $\mathcal{S G} \mathcal{S}^{k}$ & $S G S$ in $\mathcal{S G S}$ satisfying formula $(k)$ \\
\hline
\end{tabular}

Finally, note that we allow for the possibility that $A \subseteq D$. This implies that agents can reason about themselves, their properties, and relationships: some examples of the expressiveness possibilities of the present formalism are given in Section 3.2.

\subsection{Kripke Frames}

While Kripke frames are less intuitive than interpreted systems to model MAS, they are more convenient for the purpose of formal analysis, notably completeness investigations. As customarily in epistemic logic, we work with frames with equivalence relations $\sim_{i}$, that is, for a non-empty set $W$,

for every $w$ in $W, \quad w \sim_{i} w \quad$ reflexivity for every $w, w^{\prime}$ in $W, \quad w \sim_{i} w^{\prime}$ implies $w^{\prime} \sim_{i} w \quad$ symmetry for every $w, w^{\prime}, w^{\prime \prime}$ in $W, w \sim_{i} w^{\prime}$ and $w^{\prime} \sim_{i} w^{\prime \prime}$ implies $w \sim_{i} w^{\prime \prime}$ transitivity so we take the following definition:

Definition 6 (Frames) A Kripke frame, or frame, is a tuple $\mathcal{F}=\langle W$, 
$\left.\left\{\sim_{i}\right\}_{i \in A}, D,\left\{D_{i}\right\}_{i \in A}, F,\left\{F_{i}\right\}_{i \in A}\right\rangle$ such that $W$ is a non-empty set; for $i \in A$, $\sim_{i}$ is an equivalence relation on $W ; D$ is a non-empty set of individuals, and for $i \in A, D_{i}$ is a subset of $D ; F \subseteq\{f \mid f: W \rightarrow D\}$ is a non-empty set of functions from $W$ to $D$, and for $i \in A, F_{i}$ is a subset of $F$.

The class of all Kripke frames is denoted by $\mathcal{K}$.

In the model theory of modal logic $W$ is usually refered to as the set of possible worlds, while each $\sim_{i}$ is the accessibility relation between worlds [37]. The intuitive meaning of the elements $D$ and $F$ in a Kripke frame is similar to that in a system of global states: they represent the domains of interpretation for global and local terms respectively.

In contrast with interpreted systems, Kripke frames are static structures with no obvious relation to the temporal evolution of a system. However, given that also our approach to QIS is static and we will focus on purely epistemic languages, the present definition of Kripke frames is adequate with our purposes of theoretical investigations.

In the next section we consider some specializations of the notion of frame.

\subsection{Further Kripke Frames}

In order to investigate varying, regular and objectual SGS, we introduce varying, regular and objectual Kripke frames as follows:

Definition 7 (Varying frames) $A$ varying frame is a tuple $\mathcal{F}=\langle W$, $\left.\left\{\sim_{i}\right\}_{i \in A}, D,\left\{D_{i}\right\}_{i \in A}, F,\left\{F_{i}\right\}_{i \in A}\right\rangle$ such that $W, \sim_{i}, D$, and $F$ are defined as in Definition 6, and for each $i \in A, D_{i}$ is a function from $W$ to the power set $\wp(D)$, and $F_{i}$ is a function from $W$ to the power set $\wp(F)$.

We denote the class of all varying Kripke frames as $\mathcal{K}^{\text {var }}$.

In analogy with regular SGS we have also regular Kripke frames.

Definition 8 (Regular frames) A regular frame is a varying frame such that:

$$
\text { for all } w, w^{\prime} \in W, w \sim_{i} w^{\prime} \text { implies } D_{i}(w)=D_{i}\left(w^{\prime}\right) \text { and } F_{i}(w)=F_{i}\left(w^{\prime}\right)
$$

We denote the class of all regular frames as $\mathcal{K}^{\text {reg }}$.

We now introduce the objectual frames.

Definition 9 (Objectual frames) An objectual frame is a frame $\mathcal{F}=\langle W$, $\left.\left\{\sim_{i}\right\}_{i \in A}, D,\left\{D_{i}\right\}_{i \in A}, F,\left\{F_{i}\right\}_{i \in A}\right\rangle$ such that $D=F$, and for $i \in A, D_{i}=F_{i}$.

We denote the class of all objectual frames as $\mathcal{K}^{o b j}$. 
Objectual frames can be seen as tuples $\mathcal{F}=\left\langle W,\left\{\sim_{i}\right\}_{i \in A}, D,\left\{D_{i}\right\}_{i \in A}\right\rangle$.

Finally, for $2 \leq k \leq 6$ we introduce the class $\mathcal{K}^{k}$ of Kripke frames corresponding to $\mathcal{S G S}^{k}$ in a sense which will be made precise in the following section. First, consider the following constraints on Kripke frames.

for all $w, w^{\prime} \in W, w \sim_{i} w^{\prime}$ and $f(w) \in D_{i}$ implies $f\left(w^{\prime}\right) \in D_{i}$

for all $w, w^{\prime} \in W, f \in F_{i}, w \sim_{i} w^{\prime}$ and $f(w) \in D_{i}$ implies $f\left(w^{\prime}\right) \in D_{i}$

for all $w \in W, \quad f \in F_{i}, f(w) \in D_{i}$

for all $w, w^{\prime} \in W, f \in F_{i}$ and $w \sim_{i} w^{\prime}$ implies $f(w)=f\left(w^{\prime}\right)$

for all $w, w^{\prime} \in W, f \in F_{i}$ and $w \sim_{i} w^{\prime}$ implies $f(w)=f\left(w^{\prime}\right) \in D_{i}$

The meaning of formulas (8)-(12) is similar to that of formulas (2)-(6).

We now introduce 5 more classes of Kripke frames.

Definition 10 For every $k, 2 \leq k \leq 6, \mathcal{K}^{k} \subseteq \mathcal{K}$ is the class of the system of global states satisfying formula $(k+6)$.

All the classes of Kripke frames introduced so far are summarized in the following table, for $2 \leq k \leq 6$.

\begin{tabular}{|l|c|}
\hline \multicolumn{2}{|c|}{ Kripke frames } \\
\hline $\mathcal{K}$ & all \\
$\mathcal{K}^{\text {var }}$ & varying \\
$\mathcal{K}^{\text {obj }}$ & objectual \\
$\mathcal{K}^{\text {reg }}$ & regular \\
$\mathcal{K}^{k}$ & frames in $\mathcal{K}$ satisfying formula $(k+6)$ \\
\hline
\end{tabular}

We will use SGS to model concrete systems and protocols, but we rely on Kripke frames for technical results, notably the completeness proofs.

\subsection{Maps between Systems of Global States and Kripke Frames}

We explore the relationship between systems of global states and Kripke frames by means of the maps $f: \mathcal{S G S} \rightarrow \mathcal{K}$ and $g: \mathcal{K} \rightarrow \mathcal{S G S}$. By Lemma 14 below and Theorem 41 we will show that the axiomatisation of Kripke frames in Section 4 is sound and complete for the class of all SGS. Specifically, Lemma 14 states that every frame $\mathcal{F}=\left\langle W,\left\{\sim_{i}\right\}_{i \in A}, D,\left\{D_{i}\right\}_{i \in A}, F,\left\{F_{i}\right\}_{i \in A}\right\rangle$ is isomorphic to $f(g(\mathcal{F}))=\left\langle W^{\prime},\left\{\sim_{i}^{\prime}\right\}_{i \in A}, D^{\prime},\left\{D_{i}^{\prime}\right\}_{i \in A}, F^{\prime},\left\{F_{i}^{\prime}\right\}_{i \in A}\right\rangle$, that is, there are bijections between $W$ and $W^{\prime}, D$ and $D^{\prime}, F$ and $F^{\prime}$, the corresponding $D_{i}$ and $D_{i}^{\prime}$, and the corresponding $F_{i}$ and $F_{i}^{\prime}$. In addition, we can show that 
$w \sim_{i} w^{\prime}$ iff $(f \circ g)(w) \sim_{i}^{\prime}(f \circ g)\left(w^{\prime}\right)$. As a consequence, every sound and complete axiomatisation of Kripke frames is also an axiomatisation of the systems of global states.

In order to prove this result we start by introducing the map $f: \mathcal{S G S} \rightarrow \mathcal{K}$.

Definition 11 Let $\mathcal{S}=\left\langle S, D,\left\{D_{i}\right\}_{i \in A}, F,\left\{F_{i}\right\}_{i \in A}\right\rangle$ be a system of global states, define $f(\mathcal{S})$ as the tuple $\left\langle S,\left\{\sim_{i}\right\}_{i \in A}, D,\left\{D_{i}\right\}_{i \in A}, F,\left\{F_{i}\right\}_{i \in A}\right\rangle$ where for $i \in A$, each relation $\sim_{i}$ on $S$ is defined by $\left\langle l_{e}, l_{1}, \ldots, l_{n}\right\rangle \sim_{i}\left\langle l_{e}^{\prime}, l_{1}^{\prime}, \ldots, l_{n}^{\prime}\right\rangle$ iff $l_{i}=l_{i}^{\prime}$.

It is easy to check that $\sim_{i}$ is an equivalence relation and that $f(\mathcal{S})$ is a frame. To define the converse map $g: \mathcal{K} \rightarrow \mathcal{S G S}$, let $\mathcal{F}=\left\langle W,\left\{\sim_{i}\right\}_{i \in A}, D,\left\{D_{i}\right\}_{i \in A}\right.$, $\left.F,\left\{F_{i}\right\}_{i \in A}\right\rangle$ be a frame. For every equivalence relation $\sim_{i}$, for $w \in W$, we define the equivalence class $[w]_{\sim_{i}}=\left\{w^{\prime} \mid w \sim_{i} w^{\prime}\right\}$ to be a local state for agent $i$, and $w$ the local state for the environment.

Definition 12 Given a frame $\mathcal{F}=\left\langle W,\left\{\sim_{i}\right\}_{i \in A}, D,\left\{D_{i}\right\}_{i \in A}, F,\left\{F_{i}\right\}_{i \in A}\right\rangle$, let $g(\mathcal{F})$ be the tuple $\left\langle S, D,\left\{D_{i}\right\}_{i \in A}, F^{\prime},\left\{F_{i}^{\prime}\right\}_{i \in A}\right\rangle$ where $S$ contains all the $n+1$ tuples $\left\langle w,[w]_{\sim_{1}}, \ldots,[w]_{\sim_{n}}\right\rangle ; F^{\prime}$ is the set of functions $f^{\prime}$ such that $f^{\prime}\left(\left\langle w,[w]_{\sim_{1}}, \ldots,[w]_{\sim_{n}}\right\rangle\right)=f(w)$, for $f \in F$; and for $i \in A, F_{i}=\left\{f^{\prime}\right.$ $f^{\prime}\left(\left\langle w,[w]_{\sim_{1}}, \ldots,[w]_{\sim_{n}}\right\rangle\right)=f(w)$, for $\left.f \in F_{i}\right\}$.

By construction the structure $g(\mathcal{F})$ is a system of global states.

We now prove that the compositions $f \circ g$ and $g \circ f$ are isomorphisms on the respective structures. Only Lemma 14 below is actually needed to prove completeness with respect to SGS (see Section 4), but we also present Lemma 13 to state precisely the correspondence between systems of global states and Kripke frames. In what follows $\pi_{i}\left(\left\langle a_{1}, \ldots, a_{m}\right\rangle\right)=a_{i}$, for $i \leq m$.

Lemma 13 Every system of global states $\mathcal{S}$ is isomorphic to $g(f(\mathcal{S}))$.

Proof. If $\mathcal{S}=\left\langle S, D,\left\{D_{i}\right\}_{i \in A}, F,\left\{F_{i}\right\}_{i \in A}\right\rangle$ is an SGS, then $g(f(\mathcal{S}))=\left\langle S^{\prime}, D\right.$, $\left.\left\{D_{i}\right\}_{i \in A}, F^{\prime},\left\{F_{i}^{\prime}\right\}_{i \in A}\right\rangle$ is an SGS as well such that $S^{\prime}$ is the set of $n+1$-tuples $\left\langle s,[s]_{\sim_{1}}, \ldots,[s]_{\sim_{n}}\right\rangle$, for $s \in S$. The function $h(s)=\left\langle s,[s]_{\sim_{1}}, \ldots,[s]_{\sim_{n}}\right\rangle$ is a bijection between $S$ and $S^{\prime}$ : it is one-to-one as if $s, s^{\prime} \in S^{\prime}$ and $s=s^{\prime}$, then in particular $\pi_{1}(s)=\pi_{1}\left(s^{\prime}\right)$. It is onto as for $s \in S^{\prime}, \pi_{1}(s) \in S$ is such that $h\left(\pi_{1}(s)\right)=s$. Identity is trivially a bijection on $D$, and for $f \in F, f^{\prime} \in F^{\prime}$, the function $h^{\prime}$ such that $h^{\prime}(f)=f^{\prime}$ iff $f(s)=f\left(\left\langle s,[s]_{\sim_{1}}, \ldots,[s]_{\sim_{n}}\right\rangle\right)$, for $s \in S$, is a bijection between $F$ and $F^{\prime}$.

Lemma 14 Every Kripke frame $\mathcal{F}$ is isomorphic to $f(g(\mathcal{F}))$.

Proof. If $\mathcal{F}=\left\langle W,\left\{\sim_{i}\right\}_{i \in A}, D,\left\{D_{i}\right\}_{i \in A}, F,\left\{F_{i}\right\}_{i \in A}\right\rangle$ is a Kripke frame, then $f(g(\mathcal{F}))=\left\langle W^{\prime},\left\{\sim_{i}^{\prime}\right\}_{i \in A}, D,\left\{D_{i}\right\}_{i \in A}, F^{\prime},\left\{F_{i}^{\prime}\right\}_{i \in A}\right\rangle$ is such that $W^{\prime}$ is the set of $n+1$-tuples $\left\langle w,[w]_{\sim_{1}}, \ldots,[w]_{\sim_{n}}\right\rangle$, for $w \in W$. The function $h(w)=\left\langle w,[w]_{\sim_{1}}\right.$, $\left.\ldots,[w]_{\sim_{n}}\right\rangle$ is a bijection between $W$ and $W^{\prime}$ : it is one-to-one as if $w, w^{\prime} \in W^{\prime}$ and $w=w^{\prime}$, then in particular $\pi_{1}(w)=\pi_{1}\left(w^{\prime}\right)$. It is onto as for $w \in W^{\prime}$, 
$\pi_{1}(w) \in W$ is such that $h\left(\pi_{1}(w)\right)=w$. Also in this case the identity on $D$ is a bijection, and for $f \in F, f^{\prime} \in F^{\prime}$, the function $h^{\prime}$ such that $h^{\prime}(f)=$ $f^{\prime}$ iff $f(w)=f\left(\left\langle w,[w]_{\sim_{1}}, \ldots,[w]_{\sim_{n}}\right\rangle\right)$, for $w \in W$, is a bijection between $F$ and $F^{\prime}$. Moreover, $w \sim_{i} w^{\prime}$ iff $[w]_{\sim_{i}}=\left[w^{\prime}\right]_{\sim_{i}}$ iff $\left\langle w,[w]_{\sim_{1}}, \ldots,[w]_{\sim_{n}}\right\rangle \sim_{i}^{\prime}$ $\left\langle w^{\prime},\left[w^{\prime}\right]_{\sim_{1}}, \ldots,\left[w^{\prime}\right]_{\sim_{n}}\right\rangle$.

By Lemma 14 and Theorem 41 we will show that the axiomatisation of Kripke frames presented in Section 4 is adequate also for the systems of global states.

\subsection{Further Maps}

It is straightforward to extend the maps $f$ and $g$ to deal also with the other classes of SGS and Kripke frames. As regards varying SGS and frames, define map $f^{\text {var }}: \mathcal{S G S}^{\text {var }} \rightarrow \mathcal{K}^{\text {var }}$ as $f$, and define map $g^{\text {var }}: \mathcal{K}^{\text {var }} \rightarrow \mathcal{S G S}^{\text {var }}$ to be the same as $g$ except that for $s=\left\langle w,[w]_{\sim_{1}}, \ldots,[w]_{\sim_{n}}\right\rangle \in g^{\operatorname{var}}(\mathcal{F}), D_{i}^{\prime}(s)$ is defined as $D_{i}(w)$ and $F_{i}^{\prime}(s)$ is defined as $\left\{f^{\prime} \mid f^{\prime}\left(s^{\prime}\right)=f\left(w^{\prime}\right)\right.$, for $f \in F_{i}(w)$, $\left.w^{\prime}=\pi_{1}\left(s^{\prime}\right)\right\}$.

We state the following isomorphism result, the proof follows those in Lemmas 13 and 14 with little modifications.

Corollary 15 Every varying $S G S \mathcal{S}$ is isomorphic to $g^{\text {var }}\left(f^{v a r}(\mathcal{S})\right)$.

Every varying frame $\mathcal{F}$ is isomorphic to $f^{v a r}\left(g^{\text {var }}(\mathcal{F})\right)$.

Further, it can be shown that $f^{v a r}(\mathcal{S})$ is a regular frame, whenever $\mathcal{S}$ is a regular SGS. Also, $g^{\operatorname{var}}(\mathcal{F})$ is a regular SGS, whenever $\mathcal{F}$ is a regular frame. Therefore, we have the following:

Corollary 16 Every regular $S G S \mathcal{S}$ is isomorphic to $g^{\operatorname{var}}\left(f^{\text {var }}(\mathcal{S})\right)$. Every regular frame $\mathcal{F}$ is isomorphic to $f^{v a r}\left(g^{v a r}(\mathcal{F})\right)$.

The map $f^{o b j}: \mathcal{S} \mathcal{G} \mathcal{S}^{o b j} \rightarrow \mathcal{K}^{o b j}$ from objectual systems of global states to Kripke frames is defined as the restriction of $f$ to $\left\langle S, D,\left\{D_{i}\right\}_{i \in A}\right\rangle$; while the map $g^{o b j}: \mathcal{K}^{o b j} \rightarrow \mathcal{S G} \mathcal{S}^{o b j}$ is similarly defined as the restrictions of $g$ to $\left\langle W,\left\{\sim_{i}\right\}_{i \in A}, D,\left\{D_{i}\right\}_{i \in A}\right\rangle$. By considerations similar to those for Lemmas 13 and 14 it is easy to show the following:

Corollary 17 Every objectual $S G S \mathcal{S}$ is isomorphic to $g^{o b j}\left(f^{o b j}(\mathcal{S})\right)$.

Every objectual frame $\mathcal{F}$ is isomorphic to $f^{o b j}\left(g^{o b j}(\mathcal{F})\right)$.

Finally, for $2 \leq k \leq 6$, we can show that the frame $f(\mathcal{S})$ satisfies the formula $(k+6)$ whenever $\mathcal{S}$ satisfies $(k)$. Also, the SGS $g(\mathcal{F})$ satisfies the formula $(k)$ whenever $\mathcal{F}$ satisfies $(k+6)$. Therefore, the following holds:

Corollary 18 Let $2 \leq k \leq 6$. Every $S G S \mathcal{S} \in \mathcal{S G S}^{k}$ is isomorphic to 
$g(f(\mathcal{S}))$.

Every frame $\mathcal{F} \in \mathcal{K}^{k}$ is isomorphic to $f(g(\mathcal{F}))$.

In the following table we summarize the correspondences between systems of global states and Kripke frames, for $2 \leq k \leq 6$.

\begin{tabular}{|c|c|}
\hline Systems of Global States & Kripke Frames \\
\hline $\mathcal{S G S}$ & $\mathcal{K}$ \\
$\mathcal{S} \mathcal{G} \mathcal{S}^{\text {var }}$ & $\mathcal{K}^{\text {var }}$ \\
$\mathcal{S} \mathcal{G} \mathcal{S}^{\text {obj }}$ & $\mathcal{K}^{\text {obj }}$ \\
$\mathcal{S} \mathcal{G} \mathcal{S}^{\text {reg }}$ & $\mathcal{K}^{\text {reg }}$ \\
$\mathcal{S G} \mathcal{S}^{k}$ & $\mathcal{K}^{k}$ \\
\hline
\end{tabular}

In Section 4, Corollaries 15-18 will be used for proving completeness with respect to all classes of systems of global states, once obtained completeness for the corresponding class of Kripke frames.

\section{Syntax and Semantics}

In this section we introduce a family of first-order epistemic languages containing local and global terms, quantifiers, epistemic operators, as well as the identity symbol. These languages are interpreted on models based on Kripke frames. Finally, we present quantified interpreted systems, a valued version of the systems of global states.

\subsection{Syntax}

The first-order epistemic language $\mathcal{L}_{n}$ contains global variables $x_{1}, x_{2}, \ldots$, local variables $z_{1}, z_{2}, \ldots$, global constants $c_{1}, c_{2}, \ldots$, and local constants $d_{1}, d_{2}, \ldots$. Moreover, we have $n+1$-ary function symbols $f_{1}^{n+1}, f_{2}^{n+1}, \ldots$, and $n$-ary predicative constants $P_{1}^{n}, P_{2}^{n}, \ldots$, for $n \in \mathbb{N}$, the identity symbol $=$, the boolean connectives $\neg$ and $\rightarrow$, and for every $i \in A$, the indexed quantifier $\forall_{i}$, the epistemic operator $K_{i}$, and the unary predicative constant $A d m_{i}$.

Definition $19\left(\mathcal{L}_{n}\right)$ Terms and formulas in $\mathcal{L}_{n}$ are defined as follows:

$$
\begin{aligned}
& t::=x|z| c|d| f^{k}\left(t_{1}, \ldots, t_{k}\right) \\
& \phi::=P^{k}\left(t_{1}, \ldots, t_{k}\right)\left|t=t^{\prime}\right| A d m_{i}(t)|\neg \phi| \phi \rightarrow \phi^{\prime}\left|K_{i} \phi\right| \forall_{i} x \phi \mid \forall_{i} z \phi
\end{aligned}
$$


The operator $K_{i}$, for $i \in A$, is the standard epistemic modality [17]. The formula $K_{i} \phi$ intuitively means that "agent $i$ knows that $\phi$ is the case". The formula $\forall_{i} x \phi$ (respectively $\forall_{i} z \phi$ ) states that " $\phi$ is the case for all individuals in $D_{i}$ " (respectively $F_{i}$ ); whereas $A d m_{i}(t)$ means that the individual denoted by term $t$ is among the admissible individuals considered by agent $i$, that is, the individuals in $D_{i}$ and $F_{i}$. The symbols $\perp, \wedge, \vee, \leftrightarrow, \exists_{i}$ are defined from the other logical constants as standard, while metavariables $y, y^{\prime}, \ldots$ refer to the (local and global) variables in $\mathcal{L}_{n}$.

A global term $s$ is defined as:

$s::=x|c| f^{k}\left(s_{1}, \ldots, s_{k}\right)$

A term not complying with the above is said to be local. The metavariables $s, s^{\prime}, \ldots$ and $u, u^{\prime}, \ldots$ refer to global and local terms respectively; while $v, v^{\prime}, \ldots$ and $r, r^{\prime}, \ldots$ refer to closed global and local terms, where no variable appears. The symbol " $s$ " may represent either a state or a global term, the context will disambiguate.

Moreover, $t[\vec{y}]$ (resp. $\phi[\vec{y}]$ ) means that $\vec{y}=y_{1}, \ldots, y_{n}$ are all the (local and global) free variables in $t$ (resp. $\phi)$; while $t[\vec{y} / \vec{t}]$ (resp. $\phi[\vec{y} / \vec{t}]$ ) denotes the term (resp. formula) obtained by substituting simultaneously some, possibly all, the free occurrences of $y_{1}, \ldots, y_{n}$ in $t$ (resp. $\phi$ ) with $t_{1}, \ldots, t_{n}$, renaming bounded variables if necessary. We assume that local variables are to be substituted by local terms only, the same holds for global variables and terms.

We write GVar, LVar, GCon and LCon to denote the sets of global and local variables, and the sets of global and local constants in $\mathcal{L}_{n}$ respectively.

In this paper we consider two further languages, $\mathcal{L}_{n}^{o b j}$ and $\mathcal{L}_{n}^{D}$, for describing the objectual structures introduced in Section 2.

Definition $20\left(\mathcal{L}_{n}^{o b j}\right)$ The language $\mathcal{L}_{n}^{o b j}$ is the restriction of $\mathcal{L}_{n}$ to global terms. Terms and formulas in $\mathcal{L}_{n}^{\text {obj }}$ are defined as follows:

$$
\begin{aligned}
& t::=x|c| f^{k}\left(t_{1}, \ldots, t_{k}\right) \\
& \phi::=P^{k}\left(t_{1}, \ldots, t_{k}\right)\left|t=t^{\prime}\right| A d m_{i}(t)|\neg \phi| \phi \rightarrow \phi^{\prime}\left|K_{i} \phi\right| \forall_{i} x \phi
\end{aligned}
$$

Definition $21\left(\mathcal{L}_{n}^{D}\right)$ The language $\mathcal{L}_{n}^{D}$ extends $\mathcal{L}_{n}^{\text {obj }}$ by adding the distributed knowledge operator $D_{G}$, for every non-empty subset $G$ of $A$. Terms and formulas in $\mathcal{L}_{n}^{D}$ are defined as follows:

$$
\begin{aligned}
t:: & =x|c| f^{k}\left(t_{1}, \ldots, t_{k}\right) \\
\phi:: & =P^{k}\left(t_{1}, \ldots, t_{k}\right)\left|t=t^{\prime}\right| A d m_{i}(t)|\neg \phi| \phi \rightarrow \phi^{\prime}\left|K_{i} \phi\right| D_{G} \phi \mid \forall_{i} x \phi
\end{aligned}
$$


The formula $D_{G} \phi$ intuitively means that " $\phi$ is distributed knowledge among the agents in the subset $G$ of $A$ " (see [18] for details). We will show that the language $\mathcal{L}_{n}^{o b j}$ is expressive enough for the specification of objectual SGS, while $\mathcal{L}_{n}^{D}$ is useful for the application to distributed systems in Section 5 . The notions defined in this subsection for language $\mathcal{L}_{n}$ can be extended to $\mathcal{L}_{n}^{D}$ and $\mathcal{L}_{n}^{o b j}$ with the obvious changes. In this paper we do not consider extensions to common knowledge.

\subsection{Expressiveness}

This section provides some examples of the expressiveness of the first-order epistemic languages introduced in Section 3.1. First of all, in $\mathcal{L}_{n}$ we can express the agents' knowledge regarding properties and relationships among individuals. For instance, consider the following specifications:

(1) Agent $i$ knows that for every process $x$, agent $j$ knows that there exists a precondition $x^{\prime}$ considered by $i$, which has to be fulfilled in order for $x$ to be executed.

(2) Agent $i$ knows that among the inputs considered by agent $j$ there exists an $x$ for which agent $j$ does not know that every computation $x^{\prime}$ on input $x$ fails.

(3) Every successful transmission $x$ returns a code $z$, but agent $i$ does not know this code (maybe because the return code is encrypted).

These statements can be intuitively formalised as follows:

(1) $K_{i} \forall_{i} x\left(\operatorname{Process}(x) \rightarrow K_{j} \exists_{i} x^{\prime}\left(\operatorname{Precond}\left(x^{\prime}\right) \wedge\left(\operatorname{Executed}(x) \rightarrow \operatorname{Fulfil}\left(x^{\prime}\right)\right)\right)\right)$

(2) $K_{i} \exists_{j} x\left(\right.$ Input $\left.(x) \wedge \neg K_{j} \forall_{j} x^{\prime}\left(\operatorname{Comp}\left(x^{\prime}\right) \rightarrow \operatorname{Fails}\left(x, x^{\prime}\right)\right)\right)$

(3) $\forall_{i} x \exists_{i} z\left(z=\right.$ return-code $(x) \wedge \neg K_{i}(z=$ return-code $(x))$

We note the rich expressiveness of the language obtained by combining epistemic modalities with quantifiers, and the interplay between local and global terms in the third specification (but see also Section 5.1 below).

Also, observe that in this framework we can model the knowledge agents have about themselves, as pointed out in the introduction. In addition, and not discussed above, we obviously retain all the expressive power of propositional epistemic logic.

Further, in the framework of first-order epistemic logic we can express the de re/de dicto distinction, i.e., the difference between knowing of something as being so-and-so, and knowing that something is so-and-so. For instance, when we use an informal specification to say that,

as far as the security controller is concerned, every user is authorised to access the site, 
one could interpret this (hence implement it!) as either de dicto, i.e., descriptively:

(1) the security controller knows that every user is authorised to access the site, or de re, i.e., prescriptively:

(2) for every user, the security controller knows that he is authorised to access the site.

These two readings express different concepts. While these cannot be easily separated by means of a propositional language, in $\mathcal{L}_{n}$ this is promptly done as follows:

(1) $K_{\text {SecCon }} \forall_{\text {SecCon }} x($ Authorised $(x) \rightarrow \operatorname{Access}(x))$

(2) $\forall_{\text {SecCon }} x\left(\right.$ Authorised $(x) \rightarrow K_{\text {SecCon }}$ Access $\left.(x)\right)$

The difference in meaning between the two specifications is clear. For instance, the security controller not granting access to an authorised user $a$ is a violation of (2), but not of (1), if he does not regard $a$ as an authorised user.

\subsection{Kripke Models and Quantified Interpreted Systems}

We now proceed to interpret our first-order epistemic languages on the classes of Kripke frames defined in Section 2. Then we introduce quantified interpreted systems, a valued version of the systems of global states.

Definition 22 (Kripke model) A Kripke model based on a frame $\mathcal{F}$, or model, is a pair $\mathcal{M}=\langle\mathcal{F}, I\rangle$ where the interpretation $I$ is such that:

- if $c$ is a global constant, then $I(c) \in D$;

- if $d$ is a local constant, then $I(d) \in F$;

- if $f^{k}$ is a k-ary function symbol, then $I\left(f^{k}\right)$ is a $k$-ary function from $F^{k}$ to $F$ such that for $\vec{g} \in F^{k}$, we have $\left(I\left(f^{k}\right)(\vec{g})\right)(w)=I\left(f^{k}\right)\left(g_{1}(w), \ldots, g_{k}(w)\right)$;

- for $w \in W, I\left(P^{k}, w\right)$ is a k-ary relation on $D, I\left(A d m_{i}, w\right)=D_{i} \cup F_{i}$, and $I(=, w)$ is the equality relation on $D$.

In a Kripke model global constants are interpreted rigidly, while local constants are not, so it can be the case that $I(d)(w)$ is different from $I(d)\left(w^{\prime}\right)$, for $w \neq w^{\prime}$. In particular, it can be the case that $I(d)(w) \in D_{i}$ but $I(d)\left(w^{\prime}\right) \notin D_{i}$. This case models the scenario in which agent $i$ does not know whether the denotation of term $d$ is among the individuals in her domain of quantification. Shortly we will see how to deal with this situation.

Each $I\left(f^{k}\right)$ is a function from $F^{k}$ to $F$, but if the arguments are constant 
functions, i.e., elements in $D$, then $I\left(f^{k}\right)$ is a function from $D^{k}$ to $D$. The condition on $I\left(f^{k}\right)$ guarantees that identical terms can be substituted, that is, if the denotations of terms $t^{\prime}$ and $t^{\prime \prime}$ are identical, then the denotation of substitued term $t\left[y / t^{\prime}\right]$ is identical to $t\left[y / t^{\prime \prime}\right]$.

Finally, we remark that $A d m_{i}$ is an intensional predicate, i.e., its interpretation is $D_{i} \cup F_{i}$, not just a subset of $D$; this is because we consider domains of intensional objects as well as domains of individuals.

We will consider also varying, regular, and objectual models, based on varying, regular, and objectual frames.

Now let $\sigma$ be an assignment, i.e., any function from $G V$ ar to $D$ and from $L V$ ar to $F$.

Definition 23 (Valuation) The valuation $I^{\sigma}(t, w) \in D$ of a term $t$ at a world $w$ is inductively defined as follows:

$$
\begin{aligned}
& I^{\sigma}(x, w)=I^{\sigma}(x) \quad=\sigma(x) \\
& I^{\sigma}(z, w)=I^{\sigma}(z)(w)=\sigma(z)(w) \\
& I^{\sigma}(c, w)=I^{\sigma}(c) \quad=I(c) \\
& I^{\sigma}(d, w)=I^{\sigma}(d)(w)=I(d)(w) \\
& I^{\sigma}\left(f^{k}\left(t_{1}, \ldots, t_{k}\right), w\right)=I\left(f^{k}\right)\left(I^{\sigma}\left(t_{1}, w\right), \ldots, I^{\sigma}\left(t_{k}, w\right)\right) \\
& =\left(I\left(f^{k}\right)\left(I^{\sigma}\left(t_{1}\right), \ldots, I^{\sigma}\left(t_{k}\right)\right)\right)(w) \text {, by the constraint on } I\left(f^{k}\right)
\end{aligned}
$$

The definition of $I^{\sigma}(t) \in D \cup F$ can be derived from Definition 23. Note that if $t$ is a global term, then $I^{\sigma}(t)=I^{\sigma}(t, w)$, for every $w \in W$. On the other hand, if $t$ is a local term, then $I^{\sigma}(t)$ is an intensional object belonging to $F$. The valuation of terms in $\mathcal{L}_{n}^{o b j}$ is restricted to global terms.

The variant $\sigma\left(\begin{array}{l}x \\ a\end{array}\right)$ of an assignment $\sigma$ assigns $a \in D$ to $x \in G V a r$ and coincides with $\sigma$ on all the other variables; the variant $\sigma\left(\begin{array}{l}z \\ f\end{array}\right)$ for $f \in F, z \in L V$ ar is similarly defined.

Definition 24 (Satisfaction) The satisfaction relation $\models$ for $\phi \in \mathcal{L}_{n}, w \in$ $W$, and an assignment $\sigma$ is inductively defined as follows:

$$
\begin{array}{lll}
\left(\mathcal{M}^{\sigma}, w\right) \models P^{k}\left(t_{1}, \ldots, t_{k}\right) & \text { iff } & \left\langle I^{\sigma}\left(t_{1}, w\right), \ldots, I^{\sigma}\left(t_{k}, w\right)\right\rangle \in I\left(P^{k}, w\right) \\
\left(\mathcal{M}^{\sigma}, w\right) \models t=t^{\prime} & \text { iff } & I^{\sigma}(t, w)=I^{\sigma}\left(t^{\prime}, w\right) \\
\left(\mathcal{M}^{\sigma}, w\right) \models A d m_{i}(t) & \text { iff } & I^{\sigma}(t) \in D_{i} \cup F_{i} \\
\left(\mathcal{M}^{\sigma}, w\right) \models \neg \psi & \text { iff } & \left(\mathcal{M}^{\sigma}, w\right) \forall \psi \\
\left(\mathcal{M}^{\sigma}, w\right) \models \psi \rightarrow \psi^{\prime} & \text { iff } & \left(\mathcal{M}^{\sigma}, w\right) \forall \psi \text { or }\left(\mathcal{M}^{\sigma}, w\right) \models \psi^{\prime} \\
\left(\mathcal{M}^{\sigma}, w\right) \models K_{i} \psi & \text { iff } & \text { for all } w^{\prime} \in W, w \sim_{i} w^{\prime} \text { implies }\left(\mathcal{M}^{\sigma}, w^{\prime}\right) \models \psi \\
\left(\mathcal{M}^{\sigma}, w\right) \models \forall_{i} x \psi & \text { iff } & \text { for all } a \in D_{i},\left(\mathcal{M}^{\sigma\left(\begin{array}{l}
x \\
a
\end{array}\right)}, w\right) \models \psi \\
\left(\mathcal{M}^{\sigma}, w\right) \models \forall_{i} z \psi & \text { iff } & \text { for all } f \in F_{i},\left(\mathcal{M}^{\sigma\left(\begin{array}{l}
z \\
f
\end{array}\right)}, w\right) \models \psi
\end{array}
$$

The truth conditions for formulas containing $\perp, \wedge, \vee, \leftrightarrow, \exists_{i}$ can be defined from those above. The formula $A d m_{i}(t)$ means that the individual $I^{\sigma}(t) \in$ $D_{i} \cup F_{i}$ is among those admissible for agent $i$. In particular, if $t$ is a global 
term then $I^{\sigma}(t)=I^{\sigma}(t, w) \in D_{i}$. On the other hand, if $t$ is a local term then $I^{\sigma}(t)$ is an intensional object in $F_{i}$.

If we consider varying models, the definition of satisfaction is as above but for the following clauses:

Definition 25 The satisfaction relation $\models$ for $\phi \in \mathcal{L}_{n}, w \in W$, and an assignment $\sigma$ is inductively defined as follows:

$$
\begin{aligned}
& \left(\mathcal{M}^{\sigma}, w\right) \models A d m_{i}(t) \quad \text { iff } \quad I^{\sigma}(t) \in D_{i}(w) \cup F_{i}(w) \\
& \left(\mathcal{M}^{\sigma}, w\right) \models \forall_{i} x \psi \quad \text { iff } \quad \text { for all } a \in D_{i}(w),\left(\mathcal{M}^{\left.\sigma\left(\begin{array}{c}
x \\
a
\end{array}\right), w\right) \models \psi}\right. \\
& \left(\mathcal{M}^{\sigma}, w\right) \models \forall_{i} z \psi \quad \text { iff } \quad \text { for all } f \in F_{i}(w),\left(\mathcal{M}^{\sigma\left(\begin{array}{l}
z \\
f
\end{array}\right)}, w\right) \models \psi
\end{aligned}
$$

The meaning of a formula $\phi \in \mathcal{L}_{n}^{o b j}$ in an objectual model $\mathcal{M}$ is defined by restricting Definition 24 to global terms. Further, the truth conditions for $D_{G} \phi \in \mathcal{L}_{n}^{D}$ in objectual models are specified as follows:

$$
\left(\mathcal{M}^{\sigma}, w\right) \models D_{G} \psi \quad \text { iff } \quad \text { for } w^{\prime} \in W,\left(w, w^{\prime}\right) \in \bigcap_{i \in G} \sim_{i} \text { implies }\left(\mathcal{M}^{\sigma}, w^{\prime}\right) \models \psi
$$

As customary, we say that a formula $\phi$ is true at a world $w$ iff it is satisfied at $w$ by every assignment $\sigma ; \phi$ is valid on a model $\mathcal{M}$ iff it is true at every world in $\mathcal{M} ; \phi$ is valid on a frame $\mathcal{F}$ iff it is valid on every model based on $\mathcal{F} ; \phi$ is valid on a class $\mathcal{C}$ of frames iff it is valid on every frame in $\mathcal{C}$.

Let $\Delta$ be a set of formulas, $\mathcal{M}$ is a model for $\Delta$, or $\mathcal{M} \models \Delta$, iff every formula in $\Delta$ is valid on $\mathcal{M}$, and $\mathcal{F}$ is a frame for $\Delta$, or $\mathcal{F} \models \Delta$, iff every model based on $\mathcal{F}$ is a model for $\Delta$. A frame $\mathcal{F}$ for $\Delta$ will also be called $\Delta$-frame, by $\Delta$-K we denote the class of $\Delta$-frames.

We have now the formal machinery to introduce quantified interpreted systems as follows.

Definition 26 (Quantified Interpreted Systems) Given an $S G S$ S, a quantified interpreted systems $(Q I S)$ is a pair $\mathcal{P}=\langle\mathcal{S}, I\rangle$ such that $I$ is an interpretation of $\mathcal{L}_{n}$ in $f(\mathcal{S})$.

The notions of satisfaction, truth and validity on QIS can be defined as above. Let $\mathcal{P}_{f}=\langle f(\mathcal{S}), I\rangle$ be the Kripke model associated with the quantified interpreted system $\mathcal{P}=\langle\mathcal{S}, I\rangle$, then

$$
\left(\mathcal{P}^{\sigma}, s\right) \models \phi \quad \text { iff } \quad\left(\mathcal{P}_{f}^{\sigma}, s\right) \models \phi
$$

In particular, we can show that the truth conditions for epistemic modalities in interpreted systems are preserved, that is, the definition above via Kripke models boils down to the usual truth conditions in interpreted systems:

$\left(\mathcal{P}^{\sigma}, s\right) \models K_{i} \psi \quad$ iff for $s^{\prime} \in S, l_{i}(s)=l_{i}\left(s^{\prime}\right)$ implies $\left(\mathcal{P}^{\sigma}, s^{\prime}\right) \models \psi$

$\left(\mathcal{P}^{\sigma}, s\right) \models D_{G} \psi$ iff for $s^{\prime} \in S$, if for all $i \in G, l_{i}(s)=l_{i}\left(s^{\prime}\right)$, then $\left(\mathcal{P}^{\sigma}, s^{\prime}\right) \models \psi$ 
We say that a formula $\phi \in \mathcal{L}_{n}$ is valid on a quantified interpreted systems $\mathcal{P}$ iff $\phi$ is valid on $\mathcal{P}_{f}$.

Definition 26 applies also to the other classes of SGS.

Definition 27 (varying QIS) Given a varying $S G S \mathcal{S}$, a varying $Q I S$ is a pair $\mathcal{P}=\langle\mathcal{S}, I\rangle$ such that $I$ is an interpretation of $\mathcal{L}_{n}$ in $f^{\text {var }}(\mathcal{S})$.

(regular QIS) Given a regular $S G S \mathcal{S}$, a regular $Q I S$ is a pair $\mathcal{P}=\langle\mathcal{S}, I\rangle$ such that $I$ is an interpretation of $\mathcal{L}_{n}$ in $f^{\text {var }}(\mathcal{S})$.

(objectual QIS) Given an objectual $S G S \mathcal{S}$, an objectual QIS is a pair $\mathcal{P}=\langle\mathcal{S}, I\rangle$ such that $I$ is an interpretation of $\mathcal{L}_{n}^{o b j}$ in $f^{o b j}(\mathcal{S})$.

$\left(\right.$ QIS $\left.^{k}\right)$ Let $2 \leq k \leq 6$. Given an $S G S \mathcal{S} \in \mathcal{S G S}^{k}$, a $Q I S^{k}$ is a pair $\mathcal{P}=\langle\mathcal{S}, I\rangle$ such that $I$ is an interpretation of $\mathcal{L}_{n}$ in $f(\mathcal{S})$.

Quantified interpreted systems will be used in Sections 5 and 6 to model various instances of multi-agent systems. But first we discuss some important validities in these structures.

\subsection{Validities}

In Section 2 we remarked that in systems of global states the domains of quantification $D_{i}$ and $F_{i}$ are independent from global states. Therefore, both the Barcan formula and its converse [19] are valid in their indexed form on the class of all SGS:

$$
\begin{array}{rlrl}
\mathcal{S G S} & \models \forall_{i} y K_{j} \phi \rightarrow K_{j} \forall_{i} y \phi & & B F_{i-j} \\
\mathcal{S G S} & \models K_{j} \forall_{i} y \phi \rightarrow \forall_{i} y K_{j} \phi & C B F_{i-j}
\end{array}
$$

These validities state that each agent knows which are the individuals she and the other agents reason about. By $B F_{i-j}$ in all agent $j$ 's epistemic alternatives there are no more individuals in agent $i$ 's domains than $j$ presently considers, so agent $j$ can generalise her knowledge. Similarly, by $C B F_{i-j}$ in all agent $j$ 's epistemic alternatives there are no fewer individuals in agent $i$ 's domains, so agent $j$ can particularise her knowledge. When an agent can generalise and particularise her knowledge on a domain of objects, we say that she has knowledge of that domain, at least implicitly.

For the same reasons we have also that:

$\mathcal{S G S} \models A d m_{j}(t) \rightarrow K_{i} A d m_{j}(t)$

NecAdm

$\mathcal{S G S} \models \neg A d m_{j}(t) \rightarrow K_{i} \neg A d m_{j}(t)$

$N e c \neg A d m$ 
By these validities every agent knows whether any individual belongs to the domain of admissible individuals of any other agent. In the language $\mathcal{L}_{n}^{D}$ the Barcan formulas hold also for the distributed knowledge operator $D_{G}$ :

$\mathcal{S G S} \models \forall_{i} y D_{G} \phi \rightarrow D_{G} \forall_{i} y \phi$

$\mathcal{S G S} \models A d m_{j}(t) \rightarrow D_{G} A d m_{j}(t)$

$\mathcal{S G S} \models D_{G} \forall_{i} y \phi \rightarrow \forall_{i} y D_{G} \phi$

$\mathcal{S G S} \models \neg A d m_{j}(t) \rightarrow D_{G} \neg A d m_{j}(t)$

These principles are rather strong even for an external account of knowledge, in which knowledge is not explicitly possessed by agents, but rather attributed to them by an external observer. After all, we introduced different domains of quantification for expressing that each agent has only a limited access to the totality of individuals. If they know all other agents' domains as well as theirs, the whole construction seems questionable. Given this, we could turn to varying SGS, where all formulas above are not valid, as quantification domains change according to global states. But this would undermine the agents' knowledge of their own domains, something equally unwelcomed.

Our solution consists in admitting $B F_{i-j}$ and $C B F_{i-j}$ only for $i=j$. In fact, these formulas hold on the class of regular SGS:
$\mathcal{S G S}^{r e g} \models \forall_{i} y K_{i} \phi \rightarrow K_{i} \forall_{i} y \phi$
$B F_{i-i}$
$\mathcal{S G S}^{r e g} \models K_{i} \forall_{i} y \phi \rightarrow \forall_{i} y K_{i} \phi$
$C B F_{i-i}$

In this way we solve the problem of having quantification domains $D_{i}(s)$ and $F_{i}(s)$, for $i \in A$, depending also on the local states of agents other than $i$ : in regular SGS quantification domains can be considered part of each agent's local state. By restricting our attention to regular SGS satisfying the restricted versions $B F_{i-i}$ and $C B F_{i-i}$ of the Barcan formulas, we model the scenario in which agents know their respective domains of quantification, but not necessarily the other agents'.

Finally, we remark that in the present semantical framwork we admit possibilia, i.e., objects in $D$ and $F$ which do not appear in quantification domains $D_{i}$ or $F_{i}$, for some agent $i \in A$. Moreover, agents may have knowledge about these possible entities, that is, there are models in which $K_{i} P(t)$ is true at some world $w$, for some $I^{\sigma}(t, w)$ belonging to $D$, but $I^{\sigma}(t, w) \notin D_{i}$. The existence of something like possible objects is a rather debated topic in the philosophy of modal logic, see [43] and [38] for a review of classic possibilist and actualist accounts. However, in AI the introduction of possibilia can be useful to model specific scenarios. For instance, an agent $i$ may know that no horse flies, i.e., $\forall_{i} x K_{i} \neg(\operatorname{Horse}(x) \wedge F l y(x))$, and yet she studied the Greek classics and knows that Pegasus is a flying horse, i.e., $K_{i}($ Horse $($ Pegasus $) \wedge F l y($ Pegasus $))$. A solution to this puzzle consists in considering Pegasus a possible object in $D \backslash D_{i}$. 


\subsection{Individual terms and identity}

As regards identity, observe that the following formulas hold for global terms on every system of global states:

$\mathcal{S G S} \models\left(s=s^{\prime}\right) \rightarrow\left(\phi[x / s] \rightarrow \phi\left[x / s^{\prime}\right]\right)$

Subst

$\mathcal{S G S} \models\left(s=s^{\prime}\right) \rightarrow K_{i}\left(s=s^{\prime}\right)$

$K_{i} I d$

$\mathcal{S G S} \models\left(s \neq s^{\prime}\right) \rightarrow K_{i}\left(s \neq s^{\prime}\right)$

but not for local terms:

$\mathcal{S G S} \not=\left(u=u^{\prime}\right) \rightarrow\left(\phi[z / u] \rightarrow \phi\left[z / u^{\prime}\right]\right)$

$\mathcal{S G S} \not \models\left(u=u^{\prime}\right) \rightarrow K_{i}\left(u=u^{\prime}\right)$

$\mathcal{S G S} \not=\left(u \neq u^{\prime}\right) \rightarrow K_{i}\left(u \neq u^{\prime}\right)$

These (in)validities motivate the names of flexible and rigid terms given in [48]. Identical global terms can be substitued in any formula, in particular in epistemic contexts; whereas for local terms we have only:

$\mathcal{S G S} \models\left(u=u^{\prime}\right) \rightarrow\left(\phi[z / u] \rightarrow \phi\left[z / u^{\prime}\right]\right)$, for atomic $\phi$

but $\phi$ has to be different from $A d m_{i}(u)$ :

$\mathcal{S G S} \not=\left(u=u^{\prime}\right) \rightarrow\left(\operatorname{Adm}_{i}(u) \rightarrow \operatorname{Adm} m_{i}\left(u^{\prime}\right)\right)$

as $A d m_{i}$ is an intensional predicate and it can be that the individuals $I^{\sigma}(u, w)$, $I^{\sigma}\left(u^{\prime}, w\right) \in D$ are identical, the intensional object $I^{\sigma}(u)$ belongs to $F_{i}$ but $I^{\sigma}\left(u^{\prime}\right) \notin F_{i}$.

The relationship between local and global terms is particularly relevant for the present investigations. We remarked that it can be the case that agent $i$ cannot distinguish between states $s$ and $s^{\prime}$, and yet we may have that $I^{\sigma}(u)(s) \in D_{i}$ and $I^{\sigma}(u)\left(s^{\prime}\right) \notin D_{i}$, for some local term $u$. This means that in state $s$ agent $i$ is aware of the object being denoted by $u$, but she does not know that she is. This feature of the semantics can be considered unsatisfactory, but one should note that agents do not always explicitly know their domains of quantification, as it is the case in varying QIS. However, it is possible to address the point above by assuming formula (2): for every $i \in A$, for $f \in F$, if $l_{i}(s)=l_{i}\left(s^{\prime}\right)$ and $f(s) \in D_{i}$, then $f\left(s^{\prime}\right) \in D_{i}$. We can capture this constraint by means of the following formula:

$$
\exists_{i} x(d=x) \rightarrow K_{i} \exists_{i} x(d=x)
$$

We can easily check that the following correspondence holds:

$$
\mathcal{S} \models \exists_{i} x(d=x) \rightarrow K_{i} \exists_{i} x(d=x) \text { iff } \mathcal{S} \text { satisfies formula (2) }
$$


Further, we can restrict this principle to only the intensional objects contained in each $F_{i}$. According to formula (3), an agent $i$ knows the denotation of only the intensional objects in his domain of quantification. Similarly to the above, this formula can by captured by means of a formula in $\mathcal{L}_{n}$ :

$$
\forall_{i} z\left(\exists_{i} x(z=x) \rightarrow K_{i} \exists_{i} x(z=x)\right)
$$

The following correspondence can be promptly proved:

$$
\mathcal{S} \models \forall_{i} z\left(\exists_{i} x(z=x) \rightarrow K_{i} \exists_{i} x(z=x)\right) \text { iff } \mathcal{S} \text { satisfies formula (3) }
$$

Finally, we strengthen formula (14) by requiring that the denotation of every local term in the domain of agent $i$ is unconditionally an individual belonging to agent $i$ 's domain of individuals, that is, formula (4) holds. Also in this case we can provide a formal counterpart to this formula in $\mathcal{L}_{n}$,

$$
\forall_{i} z K_{i} \exists_{i} x(z=x)
$$

by noting that the following correspondence holds:

$$
\mathcal{S} \models \forall_{i} z K_{i} \exists_{i} x(z=x) \text { iff } \mathcal{S} \text { satisfies formula (4) }
$$

Moreover, we can require that each agent $i$ knows at least the denotation of the local terms in her domain of quantification. This is tantamount to formula (5), which can be captured by the following formula in $\mathcal{L}_{n}$ :

$$
\forall_{i} z\left((z=u) \rightarrow K_{i}(z=u)\right)
$$

similarly to the above, we can prove that the following correspondence holds:

$$
\mathcal{S} \models \forall_{i} z\left((z=u) \rightarrow K_{i}(z=u)\right) \text { iff } \mathcal{S} \text { satisfies formula (5) }
$$

We can combine formulas (16) and (15) in order to express formula (6), i.e., the fact each agent $i$ knows the denotation of local terms, which is an individual in her domain of quantification. We express formula (6) by means of the following formula in $\mathcal{L}_{n}$ :

$$
\forall_{i} z \exists_{i} x K_{i}(z=x)
$$

and the respective correspondence holds:

$$
\mathcal{S} \models \forall_{i} z \exists_{i} x K_{i}(z=x) \text { iff } \mathcal{S} \text { satisfies formula }(6)
$$

Note that formula (17) is the de re version of (15). According to (17) for every local term $u$, there is exactly one individual which is known by agent $i$ to be the denotation of $u$.

In the next section we will consider formulas (13)-(17) and we will prove completeness of the semantical classes introduced with respect to the relevant axiomatisations. As a summary, in the next lemma we list formulas and corresponding classes of systems of global states and Kripke frames. 
Lemma 28 In the table below, for every row, the formula in the first column is valid on all and only the SGS in the second column, and it is valid in all and only frames in the third colum.

\begin{tabular}{|c|c|c|c|}
\hline \multicolumn{2}{|c|}{ Formulas } & SGS & Frames \\
\hline & $\forall_{i} y K_{i} \phi \leftrightarrow K_{i} \forall_{i} y \phi$ & $\mathcal{S} \mathcal{G} \mathcal{S}^{\text {reg }}$ & $\mathcal{K}^{r e g}$ \\
\hline$(13)$ & $\exists_{i} x(d=x) \rightarrow K_{i} \exists_{i} x(d=x)$ & $\mathcal{S G S} \mathcal{S}^{2}$ & $\mathcal{K}^{2}$ \\
\hline$(14)$ & $\forall_{i} z\left(\exists_{i} x(z=x) \rightarrow K_{i} \exists_{i} x(z=x)\right)$ & $\mathcal{S G S} \mathcal{S}^{3}$ & $\mathcal{K}^{3}$ \\
\hline$(15)$ & $\forall_{i} z K_{i} \exists_{i} x(z=x)$ & $\mathcal{S G S} \mathcal{S}^{4}$ & $\mathcal{K}^{4}$ \\
\hline$(16)$ & $\forall_{i} z\left((z=u) \rightarrow K_{i}(z=u)\right)$ & $\mathcal{S G S} \mathcal{S}^{5}$ & $\mathcal{K}^{5}$ \\
\hline$(17)$ & $\forall_{i} z \exists_{i} x K_{i}(z=x)$ & $\mathcal{S G S} \mathcal{S}^{6}$ & $\mathcal{K}^{6}$ \\
\hline
\end{tabular}

Proof. We do not provide the details of the proof, but just note that the validity of formulas on the corresponding classes of SGS follows by the remarks earlier in this section, while the validity on Kripke frames follows by corollaries (15)-(18).

We remark that classes $\mathcal{S G} \mathcal{S}^{6}$ of systems of global states and $\mathcal{K}^{6}$ of Kripke frames are tantamount to the intersections $\mathcal{S G S}^{4} \cap \mathcal{S G S}^{5}$ and $\mathcal{K}^{4} \cap \mathcal{K}^{5}$ respectively.

\section{Axiomatisations}

In this section we introduce sound and complete axiomatisations for all the classes of SGS presented in Sections 2 and 3. We start by considering the firstorder epistemic system $Q . S 5_{n}$, then we tune this calculus to cover also varying, objectual, and regular SGS, as well as the structures satisfying formulas (2)(12). While resolution and natural deduction calculi are more suitable when dealing with automated reasoning, for the purpose of the completeness proof Hilbert-style systems are easier to handle.

\subsection{System Q.S $5_{n}$}

The system $Q . S 5_{n}$ on the language $\mathcal{L}_{n}$ is a first-order multi-modal version of the propositional system $S 5$. Hereafter we list the axioms and inference rules. We use $\Rightarrow$ to denote the inference relation between formulas.

Definition $29\left(Q . S 5_{n}\right)$ The system $Q . S 5_{n}$ on $\mathcal{L}_{n}$ contains the following schemes 
of axioms and inference rules:

\begin{tabular}{|l|l|}
\hline Taut & every instance of classic propositional tautologies \\
$K$ & $K_{i}(\phi \rightarrow \psi) \rightarrow\left(K_{i} \phi \rightarrow K_{i} \psi\right)$ \\
4 & $K_{i} \phi \rightarrow \phi$ \\
5 & $K_{i} \phi \rightarrow K_{i} K_{i} \phi$ \\
MP & $\neg K_{i} \phi \rightarrow K_{i} \neg K_{i} \phi$ \\
Nec & $\phi \rightarrow \psi, \phi \Rightarrow \psi$ \\
\hline Ex & $\forall_{i} y \phi \rightarrow\left(A d m_{i}(t) \rightarrow \phi[y / t]\right)$ \\
Gen & $\phi \rightarrow\left(A d m_{i}(t) \rightarrow \psi[y / t]\right) \Rightarrow \phi \rightarrow K_{i} y \psi$, for $y$ not free in $\phi$ \\
\hline BF $F_{i-j}$ & $\forall_{i} y K_{j} \phi \rightarrow K_{j} \forall_{i} y \phi$ \\
$C B F_{i-j}$ & $K_{j} \forall_{i} y \phi \rightarrow \forall_{i} y K_{j} \phi$ \\
\hline Id & $t=t$ \\
Func & $t=t^{\prime} \rightarrow\left(t^{\prime \prime}[y / t]=t^{\prime \prime}\left[y / t^{\prime}\right]\right)$ \\
Subst & $t=t^{\prime} \rightarrow\left(\phi[y / t] \rightarrow \phi\left[y / t^{\prime}\right]\right)$, for atomic $\phi$ \\
$K_{i} I d$ & $s=s^{\prime} \rightarrow K_{i}\left(s=s^{\prime}\right)$ \\
$K_{i} D i f$ & $s \neq s^{\prime} \rightarrow K_{i}\left(s \neq s^{\prime}\right)$ \\
\hline
\end{tabular}

The first group of postulates axiomatises the multi-modal system $S 5_{n}$. Following this, we have the free logic postulates for quantification for both global and local terms. The Barcan formulas guarantee that the domains of admissible individuals are independent from global states, as shown below. Finally, we have axioms $I d$, Func and Subst for all terms, while $K_{i} I d$ and $K_{i}$ Dif hold only for global terms. We note without proof that the axioms Subst and $K_{i} I d$ are sufficient for deriving the substitution of identical global terms in any first-order modal formula $\phi$.

We define proofs and theorems as standard: $\vdash_{Q . S 5_{n}} \phi$ means that $\phi \in \mathcal{L}_{n}$ is a theorem in Q.S $.5_{n}$. Moreover, we say that $\phi \in \mathcal{L}_{n}$ is derivable in Q.S $S 5_{n}$ from the set $\Delta$ of formulas in $\mathcal{L}_{n}$, and write $\Delta \vdash_{Q . S 5_{n}} \phi$, iff there are $\phi_{1}, \ldots, \phi_{m} \in \Delta$ such that $\vdash_{Q . S 5_{n}} \phi_{1} \wedge \ldots \wedge \phi_{m} \rightarrow \phi$.

Lemma 30 Among the theorems of $Q . S 5_{n}$ we have: 


\begin{tabular}{|l|l|l|}
\hline$N e c A d m$ & $\vdash_{Q . S 5_{n}} A d m_{i}(t) \rightarrow K_{j} A d m_{i}(t)$ & Knowledge of Admissibility \\
$N e c \neg A d m$ & $\vdash_{Q . S 5_{n}} \neg A d m_{i}(t) \rightarrow K_{j} \neg A d m_{i}(t)$ & Knowledge of Inadmissibility \\
\hline
\end{tabular}

Proof. The proof for NecAdm goes as follows:
a. $\vdash_{Q . S 5_{n}} A d m_{i}(t) \rightarrow A d m_{i}(t)$
propositional tautology
b. $\vdash_{Q . S 5_{n}} \forall_{i} y A d m_{i}(y)$
c. $\vdash_{Q . S 5_{n}} K_{j} \forall_{i} y A d m_{i}(y)$
from a by Gen
d. $\vdash_{Q . S 5_{n}} \forall_{i} y K_{j} A d m_{i}(y) \rightarrow\left(A d m_{i}(t) \rightarrow K_{j} A d m_{i}(t)\right)$ axiom $E x$
e. $\vdash_{Q . S 5_{n}} K_{j} \forall_{i} y A d m_{i}(y) \rightarrow\left(A d m_{i}(t) \rightarrow K_{j} A d m_{i}(t)\right)$ from d by $C B F_{i-j}$
f. $\vdash_{Q . S 5_{n}} A d m_{i}(t) \rightarrow K_{j} A d m_{i}(t)$
from c, e by $M P$

The proof for $N e c \neg A d m$ can be obtained similarly.

\subsection{Further Systems}

By suitably tuning the systems $Q . S 5_{n}$ we can obtain provably sound and complete axiomatisations for all classes of SGS in Sections 2 and 3. For instance, by dropping $B F_{i-j}$ and $C B F_{i-j}$, and by restricting these postulates to $i=j$, we can axiomatise varying and regular SGS respectively. Further, we can axiomatise objectual SGS by restricting $Q . S 5_{n}$ to formulas where only global terms appear. Finally, we add the axioms for distributed knowledge to Q.S $5_{n}$ in order to axiomatise the set of validities on the language $\mathcal{L}_{n}^{D}$. Hereafter we list the relevant systems.

Definition 31 (Q.S5 var $)$ The system Q.S $5_{n}^{\text {var }}$ on the language $\mathcal{L}_{n}$ is obtained from Q.S $5_{n}$ by eliminating the axioms $B F_{i-j}$ and $C B F_{i-j}$.

(Q.S5 $5_{\mathbf{n}}^{\text {reg }) ~ T h e ~ s y s t e m s ~ Q . S 5 ~} 5_{n}^{\text {reg }}$ on the language $\mathcal{L}_{n}$ is obtained from Q.S $5_{n}$ by restricting $B F_{i-j}$ and $C B F_{i-j}$ to $i=j$.

(Q.S5 $5_{\mathbf{n}}^{\text {obj }}$ ) The system Q.S $5_{n}^{\text {obj }}$ is obtained by restricting Q.S $5_{n}$ to the language $\mathcal{L}_{n}^{o b j}$.

(Q.S5 $\mathbf{n}$ ) The system $Q . S 5_{n}^{D}$ on the language $\mathcal{L}_{n}^{D}$ is obtained by extending Q.S $5_{n}^{o b j}$ with the following postulates for distributed knowledge: 


$$
\begin{array}{|l|l|}
\hline K & D_{G}(\phi \rightarrow \psi) \rightarrow\left(D_{G} \phi \rightarrow D_{G} \psi\right) \\
4 & D_{G} \phi \rightarrow \phi \\
5 & D_{G} \phi \rightarrow D_{G} D_{G} \phi \\
D 1 & D_{G} \phi \rightarrow D_{G} \neg D_{G} \phi \\
D 2 & D_{G} \phi \rightarrow K_{i} \phi \\
N e c & \phi \Rightarrow D_{G}, \text { for } G \subseteq G^{\prime} \\
\hline
\end{array}
$$

(Q.S5 $\left.\mathbf{n}_{\mathbf{n}}^{\mathbf{k}}\right)$ For $2 \leq k \leq 6$, the system $Q . S 5_{n}^{k}$ is obtained by adding formula $(k+7)$ to $Q . S 5_{n}$.

Note that formulas $N e c A d m$ and $N e c \neg A d m$ are not provable in Q.S $5_{n}^{v a r}$, while for system Q.S $5_{n}^{r e g}$ we have only restricted versions of $N e c A d m$ and $N e c \neg A d m$, for $i=j$.

\subsection{Correspondence Theory}

In this section we define a correspondence theory between properties of frames and validity of formulas at first-order. In what follows a systems $S$ is identified with the set of its theorems.

Lemma 32 Let $2 \leq k \leq 6$. In the following table, for each row, $\mathcal{F}$ is an $S$ frame, i.e., $\mathcal{F} \models S$, iff $\mathcal{F}$ belongs to the class of frames in the second column.

\begin{tabular}{|c|c|}
\hline QML Systems & Kripke frames \\
\hline$Q . S 5_{n}$ & $\mathcal{K}$ \\
$Q . S 5_{n}^{\text {var }}$ & $\mathcal{K}^{\text {var }}$ \\
$Q . S 5_{n}^{r e g}$ & $\mathcal{K}^{r e g}$ \\
$Q . S 5_{n}^{o b j}$ & $\mathcal{K}^{o b j}$ \\
$Q . S 5_{n}^{D}$ & $\mathcal{K}^{o b j}$ \\
$Q . S 5_{n}^{k}$ & $\mathcal{K}^{k}$ \\
\hline
\end{tabular}

Proof. We provide an outline of the proof by showing the right to left implications for some systems. We show that $\vdash_{S} \phi$ implies $\mathcal{F} \models \phi$, where $\mathcal{F}$ belongs to the relevant class of frames. The proof is by induction on the length of the proof of $\phi$ in $S$, axioms are the base case and inference rules are the inductive step.

Q.S5n. We can easily check that the axioms of $Q . S 5_{n}$ hold on every Kripke 
model and the inference rules preserve validity. We provide detailed proofs only for axioms $B F_{i-j}$, and $K_{i} I d$. As regards $B F_{i-j}$, assume that $\left(\mathcal{M}^{\sigma}, w\right) \models$ $\forall_{i} z K_{j} \phi$. This means that for all $f \in F_{i}$, for all $w^{\prime} \in W, w \sim_{j} w^{\prime}$ implies $\left(\mathcal{M}^{\sigma\left(\begin{array}{c}z \\ f\end{array}\right)}, w^{\prime}\right) \models \phi$. Therefore, for all $w^{\prime} \in W$, for all $f \in F_{i}, w \sim_{j} w^{\prime}$ implies $\left.\left(\mathcal{M}^{\sigma(} \begin{array}{l}z \\ f\end{array}\right), w^{\prime}\right) \models \phi$, that is, $\left(\mathcal{M}^{\sigma}, w\right) \models K_{j} \forall_{i} z \phi$.

As to $K_{i} I d$, suppose that $\left(\mathcal{M}^{\sigma}, w\right) \models s=s^{\prime}$ and $w \sim_{j} w^{\prime}$. By the first hypothesis $I^{\sigma}(s, w)=I^{\sigma}\left(s^{\prime}, w\right)$, and $I^{\sigma}\left(s, w^{\prime}\right)=I^{\sigma}\left(s^{\prime}, w^{\prime}\right)$ because $s$ and $s^{\prime}$ are global terms. Therefore $\left(\mathcal{M}^{\sigma}, w^{\prime}\right) \models s=s^{\prime}$ and $\left(\mathcal{M}^{\sigma}, w\right) \models K_{i}\left(s=s^{\prime}\right)$.

Q.S5 $\mathbf{n}_{\mathbf{n}}^{\text {var }}$. Proving that the axioms of $Q . S 5_{n}^{v a r}$ hold on every varying Kripke model, and that the inference rules preserve validity can be done similarly as in the previous case.

Q.S5 ${ }_{\mathbf{n}}^{\text {reg }}$. We show that axiom $C B F_{i-i}$ holds on every regular Kripke model; the rest may be done similarly to the case $Q . S 5_{n}$. Assume that $\left(\mathcal{M}^{\sigma}, w\right) \models$ $K_{i} \forall_{i} x \phi$. This means that for any $w^{\prime} \in W, w \sim_{i} w^{\prime}$ implies that for $a \in D_{i}\left(w^{\prime}\right)$, we have $\left(\mathcal{M}^{\sigma\left(\begin{array}{l}x \\ a\end{array}\right)}, w^{\prime}\right) \models \phi$. But since $D_{i}(w)=D_{i}\left(w^{\prime}\right)$, w $\sim_{i} w^{\prime}$ implies $\left(\mathcal{M}^{\sigma\left(\begin{array}{l}x \\ a\end{array}\right)}, w^{\prime}\right) \models \phi$ for any $a \in D\left(w^{\prime}\right)$. So, $\left(\mathcal{M}^{\sigma}, w\right) \models \forall_{i} K_{i} x \phi$.

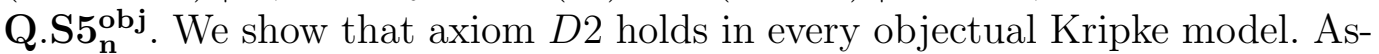
sume that $\left(\mathcal{M}^{\sigma}, w\right) \models D_{G} \phi$ and $G \subseteq G^{\prime}$. This means that for any $w, w^{\prime} \in W$, $\left(w, w^{\prime}\right) \in \bigcap_{i \in G} \sim_{i}$ implies $\left(\mathcal{M}^{\sigma}, w^{\prime}\right) \models \phi$. But $G \subseteq G^{\prime}$, so $\left(w, w^{\prime}\right) \in \bigcap_{i \in G^{\prime}} \sim_{i}$ implies $\left(\mathcal{M}^{\sigma}, w^{\prime}\right) \models \phi$. Therefore, $\left(\mathcal{M}^{\sigma}, w\right) \models D_{G^{\prime}} \phi$.

Q.S5 ${ }_{\mathbf{n}}^{\mathbf{6}}$. We show that formula (17) holds in $\mathcal{S G S}^{6}=\mathcal{S G S}^{4} \cap \mathcal{S G S}^{5}$. Assume that $f \in F_{i}$, by the constraint on $\mathcal{S G S}^{4}$ we know that there exists an $a=f(s) \in D_{i}$. Further, if $l_{i}(s)=l_{i}\left(s^{\prime}\right)$ then $f(s)=f\left(s^{\prime}\right)=a \in D_{i}$ by the constraint on $\mathcal{S G S}^{5}$. Thus, $\forall_{i} z \exists_{i} x K_{i}(z=x)$ holds.

As regards the left to right implication, we consider the cases for $Q . S 5_{n}^{1}$ and Q.S $5_{n}^{3}$.

Q.S5 $5_{\mathbf{n}}^{\mathbf{1}}$. Assume that $\mathcal{F} \models \exists_{i} x(d=x) \rightarrow K_{i} \exists_{i} x(d=x), w \sim_{i} w^{\prime}$ and $f(w) \in D_{i}$. Define a model $\mathcal{M}$ based on $\mathcal{F}$ such that $I(d)=f$. This means that $(\mathcal{M}, w) \models \exists_{i} x(d=x)$, therefore $(\mathcal{M}, w) \models K_{i} \exists_{i} x(d=x)$. Hence, $\left(\mathcal{M}, w^{\prime}\right) \models \exists_{i} x(d=x)$, which is tantamount to $f\left(w^{\prime}\right) \in D_{i}$.

Q.S5 $5_{\text {n }}^{\mathbf{3}}$. Assume that $\mathcal{F} \models \forall_{i} z K_{i} \exists_{i} x(z=x), w \in W$, and $f \in F_{i}$. Let $\mathcal{M}$ be a model based on $\mathcal{F}$, we have that $(\mathcal{M}, w) \models \forall_{i} z K_{i} \exists_{i} x(z=x)$, in particular $\left(\mathcal{M}^{\sigma}, w\right) \models \exists_{i} x(z=x)$, for $\sigma(z)=f$. Thus, there exists $a \in D_{i}$ such that $a=f(w) \in D_{i}$.

We note that a result similar to Lemma 32 can be obtained also for systems of global states by means of the maps in Section 2.5. We say that $\mathcal{P}$ is a QIS for a system $S$ iff every theorem of $S$ is valid on $\mathcal{P}$, and $\mathcal{S}$ is an SGS for $S$, or $S$-SGS, iff every model based on $\mathcal{S}$ is a model for $S$. We write $S$ - $\mathcal{S G S}$ for denoting the class of $S$-SGS.

Lemma 33 Let $2 \leq k \leq 6$. The following identities between classes of $S G S$ hold: 


$$
\begin{array}{ll}
Q . S 5_{n}-\mathcal{S G S} & =\mathcal{S G} \mathcal{S} \\
Q . S 5_{n}^{\text {var }}-\mathcal{S} \mathcal{G} \mathcal{S} & =\mathcal{S G} \mathcal{S}^{\text {var }} \\
Q . S 5_{n}^{\text {reg }}-\mathcal{S G S} & =\mathcal{S G} \mathcal{S}^{\text {reg }} \\
Q . S 5_{n}^{\text {obj }}-\mathcal{S G S} & =\mathcal{S G} \mathcal{S} \mathcal{S}^{\text {obj }} \\
Q . S 5_{n}^{D}-\mathcal{S} \mathcal{S} \mathcal{S} & =\mathcal{S G} \mathcal{S}^{\text {obj }} \\
Q . S 5_{n}^{k}-\mathcal{S} \mathcal{G} \mathcal{S} & =\mathcal{S} \mathcal{G} \mathcal{S}^{k}
\end{array}
$$

Proof. The identies above follow from Lemma 32 and the maps in Section 2.5

\subsection{Soundness}

In showing that our QML systems are sound and complete axiomatisations of the corresponding classes of system of global states we first prove soundness.

Lemma 34 (Soundness) Every QML system $S$ is sound with respect to the class of frames for $S$, therefore $S$ is sound also with respect to the class of $S G S$ for $S$.

Proof. We observe that by the right to left implication of Lemma 32 if $\vdash_{S} \phi$ then $\mathcal{F} \models \phi$, where $\mathcal{F}$ belongs to the class of $S$-frames. By the definition of validity on systems of global states, we obtain that $\vdash_{S} \phi$ implies $\mathcal{S} \models \phi$, where $\mathcal{S}$ belongs to the class of $S$-SGS.

We summarize the soundness results in the following table. Let $2 \leq k \leq 6$, for any row, the QML system in the first column is sound with respect to the corresponding class of frames in the second column, and therefore it is sound for the corresponding class of SGS in the third column.

\begin{tabular}{|c|c|c|}
\hline QML Systems & Kripke frames & Systems of global states \\
\hline$Q . S 5_{n}$ & $\mathcal{K}$ & $\mathcal{S G S}$ \\
$Q . S 5_{n}^{\text {var }}$ & $\mathcal{K}^{\text {var }}$ & $\mathcal{S G} \mathcal{S}^{\text {var }}$ \\
$Q . S 5_{n}^{\text {reg }}$ & $\mathcal{K}^{\text {reg }}$ & $\mathcal{S G \mathcal { S }}$ \\
$Q . S 5_{n}^{\text {reg }}$ & $\mathcal{K}^{o b j}$ & $\mathcal{S G} \mathcal{S}^{\text {obj }}$ \\
$Q . S 5_{n}^{D}$ & $\mathcal{K}^{o b j}$ & $\mathcal{S G \mathcal { S }}{ }^{o b j}$ \\
$Q . S 5_{n}^{k}$ & $\mathcal{K}^{k}$ & $\mathcal{S G S}$ \\
\hline
\end{tabular}




\subsection{Completeness of Q.S $5_{n}$}

This section is devoted to showing completeness for the QML systems introduced above with respect to the corresponding classes of Kripke frames. The completeness of $Q . S 5_{n}$ is proved by means of standard techniques in quantified modal logic $[19,22,33]$ suitably extended to fit in the present framework with indexed quantifiers and modalities, local terms, identity. Specifically, we adopt the particular version of canonical models given by Thomason in [62] for languages with an existence predicate. As standard, we show that if $\nvdash_{Q . S 5_{n}} \phi$ then there exists a Kripke model $\mathcal{M}$ such that $\mathcal{M} \not \models \phi$. In particular, we show that the canonical model $\mathcal{M}^{Q . S 5_{n}}$ for $Q . S 5_{n}$ to be defined below is a model according to Definition 22, it is based on a frame for Q.S $S 5_{n}$ and $\mathcal{M}^{Q . S 5_{n}} \not \models \phi$. Therefore, the following is a sufficient condition to prove completeness.

Proposition 35 If $Q . S 5_{n}$ does not prove a formula $\phi \in \mathcal{L}_{n}$, then the canonical model $\mathcal{M}^{Q . S 5_{n}}$ for $Q . S 5_{n}$ does not validate $\phi$.

This result relies on two lemmas: the saturation lemma and the truth lemma. Their proofs need the following definitions, where $L_{i} \phi$ is defined as $\neg K_{i} \neg \phi$ and $\Lambda$ is any set of formulas in $\mathcal{L}_{n}$ :

$\Lambda$ is consistent iff $\Lambda \nvdash_{Q . S 5_{n}} \perp$;

$\Lambda$ is maximal iff for every $\phi \in \mathcal{L}_{n}$, either $\phi \in \Lambda$ or $\neg \phi \in \Lambda$;

$\Lambda$ is max-cons iff $\Lambda$ is consistent and maximal;

$\Lambda$ is rich iff $\exists_{i} y \phi \in \Lambda$ implies that there is a term $t \in \mathcal{L}_{n}$ such that $A d m_{i}(t) \wedge \phi[y / t] \in \Lambda$;

$\Lambda$ is $L_{j}$-rich $\quad$ iff $\phi_{0} \wedge L_{j}\left(\phi_{1} \wedge \ldots \wedge L_{j}\left(\phi_{k} \wedge L_{j} \exists_{i} y \phi_{k+1}\right) \ldots\right) \in \Lambda$

for $y$ not free in $\phi_{0}, \ldots, \phi_{k}$ implies there is $t \in \mathcal{L}_{n}$ such that $\phi_{0} \wedge L_{j}\left(\phi_{1} \wedge \ldots \wedge L_{j}\left(\phi_{k} \wedge L_{j}\left(A d m_{i}(t) \wedge \phi_{k+1}[y / t]\right)\right) \ldots\right) \in \Lambda$,

$\Lambda$ is saturated iff $\Lambda$ is max-cons, rich and $L_{j}$-rich, for every $j \in A$.

The constraint on $L_{j}$-richness, which is due to Thomason [62], is needed in order to guarantee that the canonical model for $Q . S 5_{n}$ has a single outer domain $D$. We briefly sketch the proof of the saturation lemma and refer to [33] for details; the extension to the multi-modal case is straightforward.

Lemma 36 (Saturation lemma) If $\Delta$ is a consistent set of the formulas in $\mathcal{L}_{n}$, then it can be extended to a saturated set $\Pi$ on some expansion $\mathcal{L}_{n}^{+}$ obtained by adding to $\mathcal{L}_{n}$ an infinite set of new local constants and an infinite set of new global constants.

Proof. Let $\theta_{0}, \theta_{1}, \ldots$ be an enumeration of the formulas in $\mathcal{L}_{n}^{+}$, and let there be enumerations of the new global and local constants $m_{0}, m_{1}, \ldots$ We define by recursion a chain of sets of formulas in $\mathcal{L}_{n}^{+}$as follows: 


$$
\begin{gathered}
\Gamma_{0}= \\
\Gamma_{2 n+1}= \begin{cases}\Gamma_{2 n} \cup\left\{A d m_{i}\left(m_{2 n}\right) \wedge \theta_{n}\left[y / m_{2 n}\right]\right\} & \text { if } \Gamma_{2 n} \cup\left\{\exists y \theta_{n}\right\} \text { is consistent and } \\
\Gamma_{2 n} \notin \Gamma_{2 n} \cup\left\{\theta_{n}\right\} \text { is the first new constant; } & \text { otherwise. }\end{cases} \\
\Gamma_{2 n+2}= \begin{cases}\Gamma_{2 n+1} \cup\left\{\phi_{0} \wedge L_{j}\left(\phi_{1} \wedge \ldots \wedge L_{j}\left(\phi_{k} \wedge L_{j}\left(A d m_{i}\left(m_{2 n+1}\right) \wedge \phi_{k+1}\left[y / m_{2 n+1}\right]\right)\right) \ldots\right)\right\} \\
\text { if } \Gamma_{2 n+1} \cup\left\{\phi_{0} \wedge L_{j}\left(\phi_{1} \wedge \ldots \wedge L_{j}\left(\phi_{k} \wedge L_{j} \exists_{i} y \phi_{k+1}\right) \ldots\right)\right\} \text { is consistent } \\
\text { and } m_{2 n+1} \text { is the first variable not in } \Gamma_{2 n+1}, \phi_{1}, \ldots, \phi_{k+1} ; \\
\Gamma_{2 n+1} & \text { otherwise. }\end{cases}
\end{gathered}
$$

By induction we can prove that every $\Gamma_{n}$ is consistent. Thus, by the chain lemma $\Gamma=\bigcup_{n \in \mathbb{N}} \Gamma_{n}$ is a consistent, rich and $L_{j}$-rich set of formulas in $\mathcal{L}_{n}^{+}$, for every $j \in A$. By Lindenbaum's lemma $\Gamma$ can be extended to a saturated set $\Pi \supseteq \Delta$ in $\mathcal{L}_{n}^{+}$.

As a result, if $\nvdash_{Q . S 5_{n}} \phi$ then the set $\{\neg \phi\}$ is consistent and by the saturation lemma we obtain a saturated set $\Pi \supseteq\{\neg \phi\}$.

In order to introduce the canonical model $\mathcal{M}^{Q . S 5_{n}}$ for $Q . S 5_{n}$ we need a few more definitions. For closed global terms $v, v^{\prime}$, define $v \sim_{w} v^{\prime}$ iff $\left(v=v^{\prime}\right) \in w$. This is an equivalence relation and we take $[v]_{w}=\left\{v^{\prime} \mid v \sim_{w} v^{\prime}\right\}$ to be the equivalence class of $v$ in $w$.

The accessibility relation in $\mathcal{M}^{Q . S 5_{n}}$ is defined so that $w R_{i} w^{\prime}$ iff $\left\{\phi \mid K_{i} \phi \in\right.$ $w\} \subseteq w^{\prime}$ : by axioms $T, 4$ and 5 we can show that $R_{i}$ is an equivalence relation. Further, by axioms $K_{i} I d$ and $K_{i}$ Dif the definition of $[v]_{w}$ is provably independent from the choice of $w$ and $v$-i.e., $w R_{i} w^{\prime}$ implies $[v]_{w}=[v]_{w^{\prime}}$ - so we simply write $[v]$. We define each $D_{i}(w)$ as the set $\left\{[v] \mid A d m_{i}(v) \in w\right\}$. By $N e c A d m$ and $N e c \neg A d m$ we can show that also this definition is independent from the choice of $w$, therefore we simply write $D_{i}$.

Moreover, for every closed local term $r$ we introduce a function $f_{r}$ such that:

$$
f_{r}(w)= \begin{cases}{[v]} & \text { if there is a } v \text { such that }(r=v) \in w \\ \left\{r^{\prime} \mid\left(r^{\prime}=r\right) \in w\right\} & \text { otherwise. }\end{cases}
$$

Each $F_{i}(w)$ is the set $\left\{f_{r} \mid A d m_{i}(r) \in w\right\}$ : by $N e c A d m$ and $N e c \neg A d m$ also this definition is provably independent from the choice of $w$, so we simply write $F_{i}$.

Given these definitions, we present the canonical model for $Q . S 5_{n}$ as follows:

Definition 37 The canonical model for $Q . S 5_{n}$ on the language $\mathcal{L}_{n}$, with an expansion $\mathcal{L}_{n}^{+}$, is the tuple $\mathcal{M}^{Q . S 5_{n}}=\left\langle W,\left\{R_{i}\right\}_{i \in A}, D,\left\{D_{i}\right\}_{i \in A}, F,\left\{F_{i}\right\}_{i \in A}, I\right\rangle$ such that:

- $W$ is the set of all saturated sets of formulas in $\mathcal{L}_{n}^{+}$; 
- for $i \in A, w, w^{\prime} \in W, w R_{i} w^{\prime}$ iff $\left\{\phi \mid K_{i} \phi \in w\right\} \subseteq w^{\prime}$;

- the domain $D$ of individuals is the set $\left\{[v] \mid v \in \mathcal{L}_{n}^{+}\right\} \cup\left\{f_{r}(w) \mid r \in \mathcal{L}_{n}^{+}, w \in\right.$ $W\}$, and for each $i \in A, D_{i}=\left\{[v] \mid \operatorname{Adm}_{i}(v) \in w\right\}$;

- the domain $F$ of intensional objects is the set $\left\{f_{r} \mid r \in \mathcal{L}_{n}^{+}\right\}$, and for each $i \in A, F_{i}=\left\{f_{r} \mid A d m_{i}(r) \in w\right\}$;

- $I$ is an interpretation of $\mathcal{L}_{n}^{+}$such that:

- $I(c)=[c]$ and $I(d)=f_{d}$

- for $a_{1}, \ldots, a_{k} \in D \cup F, I\left(f^{k}\right)(\vec{a})$ is a function such that

$$
I\left(f^{k}\right)(\vec{a})(w)= \begin{cases}{\left[f^{k}(\vec{v})\right]} & \text { if every } a_{i}=\left[v_{i}\right] \\ f_{f^{k}(\vec{e})}(w) & \text { for } e_{i}=v_{i} \text { if } a_{i}=\left[v_{i}\right], \text { or } e_{i}=r_{i} \text { if } a_{i}=f_{r_{i}}\end{cases}
$$

- for $a_{1}, \ldots, a_{k} \in D,\langle\vec{a}\rangle \in I\left(P^{k}, w\right)$ iff $P^{k}(\vec{e}) \in w$, for $e_{i}=v_{i}$ and $a_{i}=\left[v_{i}\right]$, or $e_{i}=r_{i}$ and $a_{i}=f_{r_{i}}(w)$.

Note that the interpretation of functions and predicates is well defined by axioms Func and Subst. The following proposition holds by the remarks above.

Proposition 38 The canonical model $\mathcal{M}^{Q . S 5_{n}}$ for $Q . S 5_{n}$ satisfies the constraints in Definition 22, so $\mathcal{M}^{Q . S 5_{n}}$ is a Kripke model.

Proof. Assume that $\nvdash_{Q . S 5_{n}} \phi$, then the set $\{\neg \phi\}$ is consistent, so by Lemma 36 it can be extended to a saturated set of formulas in $\mathcal{L}_{n}^{+}$. Therefore, the set $W$ of possible worlds is non-empty. We remarked that by axioms $T, 4$ and 5 , each relation $R_{i}$ is reflexive, transitive and euclidean, i.e., it is an equivalence relation. Further, the domains $D$ and $F$ are non-empty by definition of $\mathcal{L}_{n}^{+}$, and each $D_{i}$ (respectively $F_{i}$ ) is trivially a subset of $D$ (respectively $F$ ). Finally, the interpretation $I$ is well-defined and satisfies the constraints on Kripke models by the remarks above.

Now let $\sigma$ be an assignment for local and global variables into $D$ and $F$ respectively, we can show the following:

Lemma 39 For every $w \in \mathcal{M}, t \in \mathcal{L}_{n}^{+}$, for $\sigma\left(y_{i}\right)=I\left(e_{i}\right)$,

$$
I^{\sigma}(t[\vec{y}], w)=I(t[\vec{y} / \vec{e}])(w)
$$

Proof. This can be shown by induction on the length of $t$. We give the proof for $t=f\left(t_{1}, \ldots, t_{k}\right), y=z$, and $e=d$ : 


$$
\begin{aligned}
I^{\sigma}(t[z], w)=I^{\sigma}\left(f\left(t_{1}[z], \ldots, t_{k}[z]\right), w\right) & =I(f)\left(I^{\sigma}\left(t_{1}[z], w\right), \ldots, I^{\sigma}\left(t_{k}[z], w\right)\right) \\
& =I(f)\left(I\left(t_{1}[z / d]\right)(w), \ldots, I\left(t_{k}[z / d]\right)(w)\right) \\
& =I(f)\left(I\left(t_{1}[z / d]\right), \ldots, I\left(t_{k}[z / d]\right)\right)(w) \\
& =I\left(f\left(t_{1}[z / d], \ldots, t_{k}[z / d]\right)\right)(w) \\
& =I\left(f\left(t_{1}, \ldots, t_{k}\right)[z / d]\right)(w) \\
& =I(t[z / d)(w)
\end{aligned}
$$

By Lemma 39 the base case of the truth lemma below follows. In the rest of this section we simply write $\mathcal{M}$ for $\mathcal{M}^{Q . S 5_{n}}$.

Lemma 40 (Truth lemma) For every $w \in \mathcal{M}, \phi \in \mathcal{L}_{n}^{+}$, for $\sigma\left(y_{i}\right)=I\left(e_{i}\right)$,

$$
\left(\mathcal{M}^{\sigma}, w\right) \models \phi[\vec{y}] \quad \text { iff } \quad \phi[\vec{y} / \vec{e}] \in w
$$

Proof. The proof is by induction on the structure of $\phi \in \mathcal{L}_{n}^{+}$.

$\phi=P^{k}\left(t_{1}, \ldots, t_{k}\right)$. By the definition of satisfaction and Lemma 39:

$$
\begin{aligned}
\left(\mathcal{M}^{\sigma}, w\right) \models P^{k}\left(t_{1}, \ldots, t_{k}\right)[\vec{y}] \quad & \text { iff }\left\langle I^{\sigma}\left(t_{1}[\vec{y}], w\right), \ldots, I^{\sigma}\left(t_{k}[\vec{y}], w\right)\right\rangle \in I\left(P^{k}, w\right) \\
\text { iff }\left\langle I\left(t_{1}[\vec{y} / \vec{e}]\right)(w), \ldots, I\left(t_{k}[\vec{y} / \vec{e}]\right)(w)\right\rangle \in I\left(P^{k}, w\right) & \text { iff } P^{k}\left(t_{1}[\vec{y} / \vec{e}], \ldots, t_{k}[\vec{y} / \vec{e}]\right) \in w
\end{aligned}
$$

$\phi=t=t^{\prime}$. Similar to the previous case.

$\phi=\neg \psi, \psi \rightarrow \psi^{\prime}$. The cases for the propositional connectives follow by the maximality and consistency of the worlds in the canonical model.

$\phi=\forall_{i} z \psi . \Leftarrow$ Assume that $\forall_{i} z \psi[z, \vec{y} / \vec{e}] \in w$ and $f$ is an individual in $F_{i}$. This means that $f=f_{r}$, for some $r$ such that $A d m_{i}(r)$, then $\psi[r, \vec{y} / \vec{e}] \in w$ by $E x$. By the induction hypothesis $\left(\mathcal{M}^{\sigma}, w\right) \models \psi[r, \vec{y}]$, therefore $\left(\mathcal{M}^{\sigma\left(\begin{array}{c}z \\ f_{r}\end{array}\right)}, w\right) \models \psi[z, \vec{y}]$. By the arbitrariness of the variant $\sigma\left(\begin{array}{c}z \\ f_{r}\end{array}\right)$ we obtain $\left(\mathcal{M}^{\sigma}, w\right) \models \forall_{i} z \psi[z, \vec{y}]$.

$\Rightarrow$ Assume that $\forall_{i} z \psi[z, \vec{y} / \vec{e}] \notin w$. Since $w$ is max-cons, $\exists_{i} z \neg \psi[z, \vec{y} / \vec{e}] \in w$. But $w$ is rich, so there exists $d \in L C$ on such that $A d m_{i}(d) \wedge \neg \psi[d, \vec{y} / \vec{e}] \in w$. This means that $f_{d} \in F_{i}$ and by induction hypothesis $\left(\mathcal{M}^{\sigma}, w\right) \not \forall \psi[d, \vec{y}]$. Thus, there is $f_{d} \in F_{i}$ such that $\left(\mathcal{M}^{\sigma\left(\begin{array}{c}z \\ f_{d}\end{array}\right)}, w\right) \forall \forall[z, \vec{y}]$, i.e., $\quad\left(\mathcal{M}^{\sigma}, w\right) \forall \forall_{i} z \psi[z, \vec{y}]$. $\phi=\forall_{i} x \psi$. The proof goes as in the previous case.

$\phi=K_{i} \psi . \Leftarrow$ Assume that $K_{i} \psi[\vec{y} / \vec{e}] \in w$ and $w R_{i} w^{\prime}$. By definition of $R_{i}$, $\psi[\vec{y} / \vec{e}] \in w^{\prime}$ and by induction hypothesis $\left(\mathcal{M}^{\sigma}, w^{\prime}\right) \models \psi[\vec{y}]$. Thus, $\left(\mathcal{M}^{\sigma}, w\right) \models$ $K_{i} \psi[\vec{y}]$.

$\Rightarrow$ Assume that $K_{i} \psi[\vec{y} / \vec{e}] \notin w$. Note that the set $\left\{\phi \mid K_{i} \phi \in w\right\} \cup\{\neg \psi[\vec{y} / \vec{e}]\}$ is consistent: if not, there would be $\phi_{1}, \ldots, \phi_{m} \in\left\{\phi \mid K_{i} \phi \in w\right\}$ such that $\vdash \wedge \phi \rightarrow \psi[\vec{y} / \vec{e}]$. By axiom $K, \vdash \wedge K_{i} \phi \rightarrow K_{i} \psi[\vec{y} / \vec{e}]$ and since $\wedge K_{i} \phi \in w$, also $K_{i} \psi[\vec{y} \mid \vec{e}] \in w$ against hypothesis. Since $\left\{\phi \mid K_{i} \phi \in w\right\} \cup\{\neg \psi[\vec{y} / \vec{e}]\}$ is consistent, we can extend it to a saturated set $w^{\prime}$ on $\mathcal{L}_{n}^{+}$(see [62] for further 
details) such that $w R_{i} w^{\prime}$ and $\left(\mathcal{M}^{\sigma}, w^{\prime}\right) \models \neg \psi[\vec{y}]$ by induction hypothesis. Hence, $\left(\mathcal{M}^{\sigma}, w\right) \not \forall K_{i} \psi[\vec{y}]$.

By the truth lemma we conclude that the canonical model $\mathcal{M}$ is a model for $Q . S 5_{n}$, based on a Kripke frame, falsifying any unprovable formula $\phi$. Thus, we state the following completeness result.

Theorem 41 (Completeness) The system Q.S $5_{n}$ is complete with respect to the class $\mathcal{K}$ of Kripke frames, i.e., $\mathcal{K} \models \phi$ implies $\vdash_{Q . S 5_{n}} \phi$.

Further, we have completeness also with respect to systems of global states. In fact, if $\nvdash_{Q . S 5_{n}} \phi$ then by Theorem 41 there exists a model $\mathcal{M}=\langle\mathcal{F}, I\rangle$ based on a frame $\mathcal{F}$, which falsifies $\phi$. Define the quantified interpreted system $\mathcal{P}$ as $\langle g(\mathcal{F}), I\rangle$ : by definition $\mathcal{P} \models \phi$ iff $\mathcal{P}_{f}=\langle f(g(\mathcal{F})), I\rangle$ models $\phi$, but by Lemma 14 the frame $f(g(\mathcal{F}))$ is isomorphic to $\mathcal{F}$. Hence, $\mathcal{P} \forall \forall$. As a consequence, the following implications hold:

$$
\mathcal{S G S} \models \phi \quad \Rightarrow \quad \mathcal{K} \models \phi \quad \Rightarrow \quad \vdash_{Q . S 5_{n}} \phi
$$

and we have a further completeness result.

Corollary 42 (Completeness) The system Q.S $5_{n}$ is complete with respect to the class $\mathcal{S G S}$ of systems of global states, i.e., $\mathcal{S G S} \models \phi$ implies $\vdash_{Q . S 5_{n}} \phi$.

By combining together the soundness and completeness theorems we obtain the main result of this section.

Corollary 43 (Soundness and Completeness) For every $\phi \in \mathcal{L}_{n}$,

$$
\mathcal{S G S} \models \phi \quad \text { iff } \quad \vdash_{Q . S 5_{n}} \phi
$$

We conclude that $Q . S 5_{n}$ is a sound and complete axiomatisation of the validities on the general class of systems of global states.

\subsection{Completeness for other axiomatisations}

We now show how to modify the techniques applied in the previous subsection to obtain completeness also for the other classes of Kripke frames and systems of global states considered. We start with varying and regular frames.

Theorem 44 (Completeness) The system Q.S $5_{n}^{\text {var }}$ is complete with respect to the class $\mathcal{K}^{\text {var }}$ of varying frames.

Proof. If a formula $\phi \in \mathcal{L}_{n}$ is not provable in $Q . S 5_{n}^{v a r}$, then we define the canonical model $\mathcal{M}^{Q . S 5_{n}^{\text {var }}}$ for Q.S $5_{n}^{\text {var }}$ as in Definition 37. Note that neither 
$N e c A d m$ nor $N e c \neg A d m$ are theorems in $Q . S 5_{n}^{v a r}$, so we cannot show that the various $D_{i}(w)=\left\{[v] \mid A d m_{i}(v) \in w\right\}$ and $F_{i}(w)=\left\{f_{r} \mid A d m_{i}(r) \in\right.$ $w\}$ are independent from the choice of $w$. However, we can prove that the canonical model is based on a varying Kripke frame. Also, Proposition 38 and Lemmas 39, 40 hold also for $\mathcal{M}^{Q . S 5_{n}^{v a r}}$, as their proofs do not require the Barcan formulas. Therefore, the canonical model $\mathcal{M}^{Q . S 5_{n}^{v a r}}$ satisfies $\neg \phi$ and we have completeness for varying frames.

Theorem 45 (Completeness) The system Q.S $5_{n}^{\text {reg }}$ is complete with respect to the class $\mathcal{K}^{\text {reg }}$ of regular frames.

Proof. As regards Q.S $5_{n}^{\text {reg }}$, we show that the canonical model $\mathcal{M}^{\text {Q.S5 }}{ }_{n}^{\text {reg }}$ defined as in Definition 37 satisfies constraint (7) on regular frames, i.e.,

- for all $w, w^{\prime} \in W, w R_{i} w^{\prime}$ implies $D_{i}(w)=D_{i}\left(w^{\prime}\right)$ and $F_{i}(w)=F_{i}\left(w^{\prime}\right)$

Assume that $w R_{i} w^{\prime}$ and $A d m_{i}(v) \in w$. By the restricted version $C B F_{i-i}$ of the converse of the Barcan formula we can show that $A d m_{i}(v) \rightarrow K_{i} A d m_{i}(v) \in w$, and by definition of $R_{i}$ also $A d m_{i}(v) \in w^{\prime}$. Thus, $D_{i}(w) \subseteq D_{i}\left(w^{\prime}\right)$. The other direction of the inclusion follows by symmetry of $R_{i}$. The proof of $F_{i}(w)=$ $F_{i}\left(w^{\prime}\right)$ is similar. Further, Proposition 38 and Lemmas 39, 40 hold also for $\mathcal{M}^{Q . S 5_{n}^{r e g}}$, therefore we have completeness for regular frames.

By axiomatising the class of regular SGS we showed that the restricted versions of the Barcan formulas considered are powerful enough to characterise the idea of quantification domains as part of agents' local state.

We now consider the completeness results for systems Q.S $5_{n}^{\text {obj }}$ and $Q . S 5_{n}^{D}$ with respect to objectual frames. Let $\phi \in \mathcal{L}_{n}^{o b j}$ be a formula not provable in $Q . S 5_{n}^{o b j}$, we define the canonical model for $Q . S 5_{n}^{o b j}$ as the restriction of $\mathcal{M}^{Q . S 5_{n}}$ to constant terms.

Definition 46 The canonical model for $Q . S 5_{n}^{o b j}$ on the language $\mathcal{L}_{n}^{o b j}$, with an expansion $\mathcal{L}_{n}^{o b j+}$, is the tuple $\mathcal{M}^{Q . S 5_{n}^{o b j}}=\left\langle W,\left\{R_{i}\right\}_{i \in A}, D,\left\{D_{i}\right\}_{i \in A}, I\right\rangle$ such that:

- $W$ is the set of all saturated sets of formulas in $\mathcal{L}_{n}^{\text {obj+; }}$;

- for $i \in A, R_{i}$ and $D_{i}$ are defined as in Definition 37;

- $D=\left\{[v] \mid v \in \mathcal{L}_{n}^{o b j+}\right\}$;

- I is an interpretation of $\mathcal{L}_{n}^{o b j+}$ such that:

- $I(c)=[c]$;

- for $a_{1}, \ldots, a_{k} \in D, I\left(f^{k}\right)(\vec{a})=\left[f^{k}(\vec{v})\right]$, for $a_{i}=\left[v_{i}\right]$;

- for $a_{1}, \ldots, a_{k} \in D,\langle\vec{a}\rangle \in I\left(P^{k}, w\right)$ iff $P^{k}(\vec{v}) \in w$, for $a_{i}=\left[v_{i}\right]$.

Proposition 38 and Lemmas 39 and 40 hold also for $\mathcal{M}^{Q . S 5_{n}^{o b j}}$ : the proof is similar to those presented earlier. It follows that the canonical model $\mathcal{M}^{Q . S 5_{n}^{o b j}}$ satisfies $\neg \phi$, so we have completeness for objectual frames: 
Theorem 47 (Completeness) The system Q.S $5_{n}^{\text {obj }}$ is complete with respect to the class $\mathcal{K}^{o b j}$ of objectual frames.

As regards the system $Q . S 5_{n}^{D}$, we extend to first-order the completeness proof in [18] for proposional systems with distributed knowledge consisting in unravelling the canonical model to obtain a model which is a tree. Note that an independent completeness proof for these systems appeared in [31].

First, we consider the following definition of canonical model, where $\wp^{+}(A)$ is the set of non-empty subsets of $A$ :

Definition 48 The canonical model for $Q . S 5_{n}^{D}$ on the language $\mathcal{L}_{n}^{D}$, with an expansion $\mathcal{L}_{n}^{D+}$, is the tuple $\mathcal{M}^{Q . S 5_{n}^{D}}=\left\langle W,\left\{R_{l}\right\}_{l \in A \cup \wp^{+}(A)}, D,\left\{D_{i}\right\}_{i \in A}, I\right\rangle$ such that:

- $W$ is the set of saturated sets of formulas in $\mathcal{L}_{n}^{D+}$;

- for $i \in A, R_{i}, D_{i}$, and $D, I$ are defined as in Definition 46;

- for $G \in \wp^{+}(A), w R_{G} w^{\prime}$ iff $\left\{\phi \mid D_{G} \phi \in w\right\} \subseteq w^{\prime}$.

Lemma 36 holds also for the system $Q . S 5_{n}^{D}$. Therefore, if $\forall_{Q . S 5_{n}^{D}} \phi$ there exists a saturated set $w \supseteq\{\neg \phi\}$, so the set $W$ of possible worlds in $\mathcal{M}^{Q . S 5_{n}^{D}}$ is non-empty. By using axioms $T, 4$ and 5 we can show that the various $R_{G}$ are equivalence relations. Moreover, by axioms $D 1$ and $D 2$ it follows that $R_{\{i\}}$ is equal to $R_{i}$ and $R_{G} \subseteq \bigcap_{i \in G} R_{i}$. However, in general $R_{G} \neq \bigcap_{i \in G} R_{i}$ [18]. This remark gives the rationale for introducing the pseudo-satisfaction relation $\models^{p}$, defined as $\models$ but for the distributed knowledge operator $D_{G}$ (in what follows we simply write $\mathcal{M}$ for $\left.\mathcal{M}^{Q . S 5_{n}^{D}}\right)$ :

$\left(\mathcal{M}^{\sigma}, w\right) \models^{p} D_{G} \psi \quad$ iff $\quad$ for every $w^{\prime} \in W, w R_{G} w^{\prime}$ implies $\left(\mathcal{M}^{\sigma}, w^{\prime}\right) \models^{p} \psi$

We state the truth lemma for the pseudo-satisfaction relation $\models^{p}$. The proof follows the one for Lemma 40, the distributed knowledge operator $D_{G}$ being treated as the epistemic operator $K_{i}$.

Lemma 49 (Truth lemma) For $w \in \mathcal{M}, \psi \in \mathcal{L}_{n}^{D+}$, for $\sigma\left(x_{i}\right)=\left[v_{i}\right]$,

$$
\left(\mathcal{M}^{\sigma}, w\right) \models^{p} \psi[\vec{x}] \quad \text { iff } \quad \psi[\vec{x} / \vec{v}] \in w
$$

We now show that the techniques in [18] can be extended to first-order so that $\mathcal{M}$ can be unwound into a structure $\mathcal{M}^{\prime}$ satisfying the same formulas.

In order to introduce $\mathcal{M}^{\prime}$ we need a few more definitions. Let $w, w^{\prime}$ be worlds in $W$, a path from $w$ to $w^{\prime}$ is a sequence $\left\langle w_{1}, l_{1}, w_{2}, l_{2}, \ldots, l_{k-1}, w_{k}\right\rangle$ such that:

(1) $w=w_{1}$ and $w^{\prime}=w_{k}$;

(2) $w_{1}, \ldots, w_{k} \in W$;

(3) each $l_{j}$ is either an agent or a set of agents;

(4) $\left\langle w_{j}, w_{j+1}\right\rangle \in R_{l_{j}}$. 
The reduction of a path $\left\langle w_{1}, i_{1}, w_{2}, i_{2}, \ldots, i_{k-1}, w_{k}\right\rangle$ is obtained by replacing each maximal consecutive subsequence $\left\langle w_{q}, i_{q}, w_{q+1}, i_{q+1}, \ldots, i_{r-1}, w_{r}\right\rangle$ where $i_{q}=i_{q+1}=\ldots=i_{r-1}$ by $\left\langle w_{q}, i_{q}, w_{r}\right\rangle$. A path is said to be reduced is it is equal to its reduction.

Given the canonical model $\mathcal{M}=\left\langle W,\left\{R_{l}\right\}_{l \in A \cup \wp^{+}(A)}, D,\left\{D_{i}\right\}_{i \in A}, I\right\rangle$ we define a structure $\mathcal{M}^{*}=\left\langle W^{*},\left\{R_{l}^{*}\right\}_{l \in A \cup \wp^{+}(A)}, D^{*},\left\{D_{i}^{*}\right\}_{i \in A}, I^{*}\right\rangle$ and a surjective function $h: W^{*} \rightarrow W$ such that (i) $\mathcal{M}^{*}$ is a tree, that is, for all $w, w^{\prime} \in W^{*}$, there is at most one reduced path from $w$ to $w^{\prime}$; (ii) $w R_{i}^{*} w^{\prime}$ implies $h(w) R_{i} h\left(w^{\prime}\right)$; (iii) $w R_{G}^{*} w^{\prime}$ implies $h(w) R_{G} h\left(w^{\prime}\right)$; (iv) $\left\langle a_{1}, \ldots, a_{k}\right\rangle \in I^{*}\left(P^{k}, w\right)$ iff $\left\langle a_{1}, \ldots, a_{k}\right\rangle \in I\left(P^{k}, h(w)\right)$.

The set of possible worlds $W^{*}$ is defined by induction. Let $W_{1}^{*}$ be $W$, and define $W_{k+1}^{*}$ as the set of worlds $v_{w, l, w^{\prime}}$ such that $w \in W_{k}^{*}, w^{\prime} \in W$ and $l$ is an agent or group of agents. Let $W^{*}=\bigcup_{k \in \mathbb{N}} W_{k}^{*}$, then define $h: W^{*} \rightarrow W$ by letting $h(w)=w$, for $w \in W_{1}^{*}$ and $h\left(v_{w, l, w^{\prime}}\right)=w^{\prime}$, for $w \in W_{k}^{*}$. Further, $R_{l}^{*}$ is the reflexive, symmetric and transitive closure of the relation defined for $w, w^{\prime} \in W^{*}$ if $w^{\prime}=v_{w, l, w^{\prime \prime}}$, for some $w^{\prime \prime} \in W$, and $h(w) R_{l} h\left(w^{\prime}\right)$. Finally, $I^{*}\left(P^{k}, w\right)=I\left(P^{k}, h(w)\right)$. We refer to [18] for a proof that $\mathcal{M}^{*}$ and $h$ satisfy (i)-(iv) above. In particular, we can show what follows:

Lemma 50 For $w \in W^{*}, \psi \in \mathcal{L}_{n}^{D+}$, we have

$$
\left(\mathcal{M}^{* \sigma}, w\right) \models^{p} \psi \quad \text { iff } \quad\left(\mathcal{M}^{\sigma}, h(w)\right) \models^{p} \psi
$$

Finally, we make use of the structure $\mathcal{M}^{*}$ to define a Kripke model $\mathcal{M}^{\prime}$ that falsifies the unprovable formula $\phi$.

Definition 51 The model $\mathcal{M}^{\prime}=\left\langle W^{\prime},\left\{R_{i}^{\prime}\right\}_{i \in A}, D^{\prime},\left\{D_{i}^{\prime}\right\}_{i \in A}, I^{\prime}\right\rangle$ is defined as follows:

- $W^{\prime}=W^{*}, D^{\prime}=D^{*}, D_{i}^{\prime}=D_{i}^{*}$, and $I^{\prime}=I^{*}$;

- $R_{i}^{\prime}$ is the transitive closure of $R_{i}^{*} \cup \bigcup_{i \in G} R_{G}^{*}$.

Since the various $R_{i}^{*}$ and $R_{G}^{*}$ are reflexive, transitive and symmetric, $R_{i}^{\prime}$ is an equivalence relation. We state the following result about $\mathcal{M}^{\prime}$ and refer to [18] for further details.

Lemma 52 For $w \in W^{\prime}, \psi \in \mathcal{L}_{n}^{D+}$, the following holds

$$
\left(\mathcal{M}^{\prime \sigma}, w\right) \models \psi \quad \text { iff } \quad\left(\mathcal{M}^{* \sigma}, w\right) \models^{p} \psi
$$

In conclusion, if $\phi \in \mathcal{L}_{n}^{D}$ is not provable in $Q . S 5_{n}^{D}$, then the canonical model $\mathcal{M}^{Q . S 5_{n}^{D}}$ pseudo-satisfies $\neg \phi$ by Lemma 49. By Lemma 50 also $\mathcal{M}^{*}$ pseudosatisfies $\neg \phi$, and by the result above $\mathcal{M}^{\prime}$ does not validate $\phi$. Hence, we have the following completeness result.

Theorem 53 (Completeness) The system Q.S $5_{n}^{D}$ is complete with respect 
to the class of objectual frames.

Finally, we show that formulas (13)-(17) are sufficient to guarantee that the canonical models for the systems $Q . S 5_{n}^{k}$, for $2 \leq k \leq 6$, defined as in Definition 37 , satisfy the corresponding constraints.

Theorem 54 (Completeness) For $2 \leq k \leq 6$, the system Q.S $5_{n}^{k}$ is complete with respect to the class $\mathcal{K}^{k}$ of Kripke frames.

Proof. We show that the canonical models for the systems Q.S $5_{n}^{k}$, for $2 \leq$ $k \leq 6$, satisfy the respective constraint on the classes $\mathcal{K}^{k}$ of Kripke frames.

Q.S5 $5_{\mathbf{n}}^{1}$. Assume that $w \sim_{i} w^{\prime}$ and $f(w) \in D_{i}$. If $f \in F$ then $f=f_{r}$ for some closed local term $r$, and by hypothesis $f_{r}(w)=[v]$, for some $v$ such that $A d m_{i}(v) \in w$. This means that $(r=v) \in w$, therefore $\exists x_{i}(r=x) \in w^{\prime}$ by formula (13). By richness of $w^{\prime}$ there exists $v^{\prime}$ such that $A d m_{i}\left(v^{\prime}\right) \in w^{\prime}$ and $\left(r=v^{\prime}\right) \in w^{\prime}$. Thus, $f_{r}\left(w^{\prime}\right) \in D_{i}$.

Q.S5 $5_{\mathbf{n}}^{2}$. This can be shown as above, but this time $f=f_{r}$, for some closed local term $r$, belongs to $F_{i}$.

Q.S5 $5_{\mathbf{n}}^{\mathbf{3}}$. Assume that $f=f_{r} \in F_{i}$ for some closed local term $r$, then $K_{i} \exists_{i} x(r=$ $x) \in w$ by formula (15), and in particular $\exists_{i} x(r=x) \in w$. By the richness of $w$ there exists $v$ such that $A d m_{i}(v) \in w$ and $(r=v) \in w$. Therefore, there exists $[v] \in D_{i}$ such that and $f_{r}(w)=[v]$.

Q.S5 $\mathbf{n}_{\mathbf{n}}$. Assume that $f=f_{r}$ for some closed local term $r$ belongs to $F_{i}, f_{r}(w)=$ $a \in D$, and $w \sim w^{\prime}$. By hypothesis there exists a closed term $t$ such that $I(t)=a \in D$ and $(r=t) \in w$. By formula (16) this implies that $K_{i}(r=t) \in w$ and $(r=t) \in w^{\prime}$. Thus $f_{r}(w)=f_{r}\left(w^{\prime}\right)$.

Q.S5 $\mathbf{5}$. Since formula (17) is equivalent to the conjuntion of formulas (15) and (16), we can prove that for $f \in F_{i}, w \sim_{i} w^{\prime}$ implies $f(w)=f\left(w^{\prime}\right) \in D_{i}$.

As a result, we have completeness with respect to all the relevant classes of frames.

As for system Q.S $5_{n}$, for every QML system $S$ from completeness with respect to Kripke frames we can derive completeness for systems of global state. In fact, if $\nvdash_{S} \phi$ then by Kripke completeness there exists a model $\mathcal{M}=\langle\mathcal{F}, I\rangle$ based on a $S$-frame $\mathcal{F}$, which falsifies $\phi$. Define the quantified interpreted system $\mathcal{P}$ as $\langle\gamma(\mathcal{F}), I\rangle$, where $\gamma$ is the relevant map from $S$-frames to SGS for $S$. By definition $\mathcal{P} \models \phi$ iff $\mathcal{P}_{\varphi}=\langle\varphi(\gamma(\mathcal{F})), I\rangle$ models $\phi$, where $\varphi$ is the relevant map from SGS for $S$ to $S$-frames. By the results in Section 2.6, the frame $\varphi(\gamma(\mathcal{F}))$ is isomorphic to $\mathcal{F}$. Hence, $\mathcal{P} \forall \phi$. Thus, the following implications hold: 


$$
S \text {-SGS } \models \phi \quad \Rightarrow \quad S-\mathcal{K} \models \phi \quad \Rightarrow \quad \vdash_{S} \phi
$$

and we have a further completeness result.

Corollary 55 (Completeness) Every QML system $S$ is complete with respect to the class $S-\mathcal{S G S}$ of the systems of global states for $S$, that is, $S$ $\mathcal{S G S} \models \phi$ implies $\vdash_{S} \phi$.

By combining together the soundness and completeness theorems we obtain the main result of this section.

Corollary 56 (Soundness and Completeness) For every formula $\phi$ in the language of $S$,

$$
S-\mathcal{S G S} \models \phi \quad \text { iff } \quad \vdash_{S} \phi
$$

We conclude that our QML systems are sound and complete axiomatisations of the validities on the corresponding classes of systems of global states. The corollary below summarizes the main formal results of this paper.

Corollary 57 Let $2 \leq k \leq 6$, for every row, the QML system in the first column of the following table is a sound and complete axiomatisation with

\begin{tabular}{|c|c|c|}
\hline QML Systems & Kripke frames & Systems of global states \\
\hline$Q . S 5_{n}$ & $\mathcal{K}$ & $\mathcal{S G S}$ \\
\hline$Q . S 5_{n}^{v a r}$ & $\mathcal{K}^{v a r}$ & $\mathcal{S} \mathcal{G} \mathcal{S}^{\text {var }}$ \\
\hline$Q . S 5_{n}^{r e g}$ & $\mathcal{K}^{r e g}$ & $\mathcal{S} \mathcal{G} \mathcal{S}^{r e g}$ \\
\hline$Q . S 5_{n}^{o b j}$ & $\mathcal{K}^{o b j}$ & $\mathcal{S} \mathcal{G} \mathcal{S}^{o b j}$ \\
\hline$Q . S 5_{n}^{D}$ & $\mathcal{K}^{o b j}$ & $\mathcal{S} \mathcal{G} \mathcal{S}^{o b j}$ \\
\hline$Q . S 5_{n}^{k}$ & $\mathcal{K}^{k}$ & $\mathcal{S} \mathcal{G S}^{k}$ \\
\hline
\end{tabular}
respect to the corresponding class of Kripke frames and systems of global states:

\section{Applications to Multi-Agent Systems}

In this section we show how simple MAS scenarios can be modeled by means of quantified interpreted systems. Specifically, in Section 5.1 we analyse the scenario of battlefield, and in Section 5.2 we consider an infinite version of the muddy children puzzle. Section 6 deals with a more complex application: a formal analysis of message passing systems. We maintain that these investigations show some of the advantages of first-order formalisms. 


\subsection{Battlefield}

In this subsection we model the popular game of battlefield as a quantified interpreted system, then we describe it by means of the language $\mathcal{L}_{n}$, for $n=4$. We start by considering a set of agents $A=\{1,2,3,4\}$, and we assume that each agent is assigned a quadrant in the region $\mathbb{Z} \times \mathbb{Z}$ as domain of quantification:

$$
\begin{array}{llll}
D_{4}=\{(x, y) & \left.x \in \mathbb{Z}^{-}, y \in \mathbb{Z}^{+}\right\} & D_{1}=\left\{(x, y) \mid x, y \in \mathbb{Z}^{+}\right\} \\
D_{3}=\{(x, y) & \left.x, y \in \mathbb{Z}^{-}\right\} & D_{2}=\{(x, y) & \left.x \in \mathbb{Z}^{+}, y \in \mathbb{Z}^{-}\right\}
\end{array}
$$

Intuitively, the set $D_{i}$ represents the country of agent $i$. Note that, even though in the battlefield game only a finite subset of $\mathbb{Z} \times \mathbb{Z}$ is considered, in several scenarios either we are not able, or do not want, to bound in advance the size of the game board. Thus, it can be useful to introduce a potentially infinite playing board, even though at each step only a finite portion is considered.

We assume that each agent has 5 military units, whose positions are recorded in his local state. Further, we consider couples $(x, y) \in D_{i}$ and triples $(k, x, y)$, for $1 \leq k \leq 5$, to express that there is a military unit at $(x, y)$ and that the $k$-th military unit is at $(x, y)$ respectively.

The local state $l_{i}$ of agent $i$ is defined as a 4 -tuple $\left\langle\alpha_{1}, \alpha_{2}, \alpha_{3}, \alpha_{4}\right\rangle$ such that:

- $\alpha_{i}$ is a 5 -tuple $\left\langle\left(1, x_{1}, y_{1}\right), \ldots,\left(5, x_{5}, y_{5}\right)\right\rangle$ recording the positions of agent $i$ 's units.

- for $j \neq i, \alpha_{j}$ is a possibly empty sequence of triples $(k, x, y)$ and couples $(x, y)$ recording the positions and identities of agent $i$ 's enemies' units.

Also, $l_{e}$ is the local state of the environment recording the positions and identities of all units in similar fashion.

The set $S$ contains the global states $s=\left\langle l_{e}, l_{1}, l_{2}, l_{3}, l_{4}\right\rangle$ such that if either $(x, y) \in \alpha_{j}\left(l_{i}\right)$ or $(k, x, y) \in \alpha_{j}\left(l_{i}\right)$, then $(k, x, y) \in \alpha_{i}\left(l_{i}\right)$. So, an agent may not know the position or the identity of an enemy unit, but if she does, then she cannot be wrong.

Each domain $F_{i}$ of intensional objects contains the military units $m u_{i . k}$, for $1 \leq k \leq 5$, such that $m u_{i . k}(s)=(x, y)$ iff the triple $(k, x, y)$ belongs to the component $\alpha_{i}$ of the local state $l_{i}(s)$. Moreover, we assume that our language has global and local constants for denoting the individuals in the various $D_{i}$ and $F_{i}$. We use the same notation for syntactic and semantic elements as the former mirror the latter, the context will disambiguate. Finally, let $D$ contain the real numbers, i.e., $D \supseteq \mathbb{R}$.

The quantified interpreted system $\mathcal{P}$ contains all the global states consistent with the definitions above. In particular, suppose that the initial state $s=\left\langle l_{e}, l_{1}, l_{2}, l_{3}, l_{4}\right\rangle$, describing the position of the military units at the beginning, is defined as follows:

$l_{1}(s)=\langle\langle(1,2,2),(2,6,5),(3,2,7),(4,4,12),(5,7,9)\rangle,\langle\rangle,\langle\rangle,\langle\rangle\rangle$ 
$l_{2}(s)=\langle\langle\rangle,\langle(1,3,-3),(2,7,-2),(3,6,-5),(4,3,-6),(5,8,-9)\rangle,\langle\rangle,\langle\rangle\rangle$

$l_{3}(s)=\langle\langle\rangle,\langle\rangle,\langle(1,-3,-3),(2,-3,-6),(3,-6,-3),(4,-6,-8),(5,-8,-5)\rangle,\langle\rangle\rangle$

$l_{4}(s)=\langle\langle\rangle,\langle\rangle,\langle\rangle,\langle(1,-4,4),(2,-3,9),(3,-7,7),(4,-5,12),(5,-8,11)\rangle\rangle$

The QIS $\mathcal{P}$ containing $s$ describes a situation in which the first military unit of agent 1 is positioned at $(2,2)$. In particular, agent 1 knows this fact:

$(\mathcal{P}, s) \models K_{1} \exists_{1} z(z=(2,2)) \quad$ and also $\quad(\mathcal{P}, s) \models \exists_{1} z K_{1}(z=(2,2))$

while agent 3 does not know this:

$(\mathcal{P}, s) \models \neg \exists_{1} z K_{3}(z=(2,2)) \quad$ and also $\quad(\mathcal{P}, s) \models \neg K_{3} \exists_{1} z(z=(2,2))$

Thus, agent 1 has not only de dicto knowledge that one of his military units is at $(2,2)$, but he also has de re knowledge of the first of his units being at $(2,2)$.

Consider now a function dist returning the distance between two points in $\mathbb{Z} \times \mathbb{Z}$ as a real number:

$$
I(d i s t)\left((x, y),\left(x^{\prime}, y^{\prime}\right)\right)=\sqrt{\left(x-x^{\prime}\right)^{2}+\left(y-y^{\prime}\right)^{2}}
$$

We can check that agent 2 starts with all his military units within a distance of less than 8. Further, he knows this fact, but agent 4 does not. To express this, we introduce a relation symbol $<$ with the obvious interpretation "less than".

$$
(\mathcal{P}, s) \models \forall_{2} z, z^{\prime} K_{2}\left(\operatorname{dist}\left(z, z^{\prime}\right)<8\right) \quad \text { but } \quad(\mathcal{P}, s) \not \forall \forall_{2} z, z^{\prime} K_{4}\left(\operatorname{dist}\left(z, z^{\prime}\right)<8\right)
$$

The latter formula fails as agent 4's local state is consistent with a different state $s^{\prime \prime \prime}$ such that $l_{2}\left(s^{\prime \prime \prime}\right)$ contains two military units at a distance greater than 8 , that is, $l_{4}\left(s^{\prime \prime \prime}\right)=l_{4}(s)$ but for instance

$$
l_{2}\left(s^{\prime \prime \prime}\right)=\langle\langle\rangle,\langle(1, \mathbf{3 0},-\mathbf{3 0}),(2,7,-2),(3,6,-5),(4,3,-6),(5,8,-9)\rangle,\langle\rangle,\langle\rangle\rangle
$$

Note that $s^{\prime \prime \prime} \in \mathcal{P}$ by definition of $\mathcal{P}$.

Further, consider a global constant firedist representing the maximum range of fire of the military units and set it to 8 , i.e., $I$ (firedist) $=8$. We can express the fact that the units $z$ and $z^{\prime}$ are within fire range by the following formula:

$$
\operatorname{FDist}\left(z, z^{\prime}\right)::=\operatorname{dist}\left(z, z^{\prime}\right)<\text { firedist }
$$

The following expression specifies that in the present QIS every agent has at least one unit which is not within the fire range of any other enemy unit:

$$
\left(\mathcal{P}^{\sigma}, s\right) \models \exists_{i} z \forall_{j} z^{\prime} \neg F \operatorname{Dist}\left(z, z^{\prime}\right), \text { for } j \neq i
$$

Now suppose that hostilities break out in our scenario and agent 3 somehow acquires the knowledge that one of agent 1's military units is at $(2,2)$. The 
resulting state $s^{\prime}$ differs from $s$ only for the local state of agent 3 :

$l_{3}\left(s^{\prime}\right)=\langle\langle(2,2)\rangle,\langle\rangle,\langle(1,-3,-3),(2,-3,-6),(3,-6,-3),(4,-6,-8),(5,-8,-5)\rangle,\langle\rangle\rangle$

As a consequence, agent 3 acquires de dicto knowledge that the first of his units is within the range of enemy fire, even if she does not know this fact de re, that is, she does not know which of agent 1's units actually threathens agent 3's first unit.

$\left(\mathcal{P}, s^{\prime}\right) \models \exists_{3} z K_{3} \exists_{1} z^{\prime} F \operatorname{Dist}\left(z, z^{\prime}\right) \quad$ but $\quad\left(\mathcal{P}, s^{\prime}\right) \not \exists_{3} z \exists_{1} z^{\prime} K_{3} F \operatorname{Dist}\left(z, z^{\prime}\right)$

This is because the following local states for agent 1 is consistent with agent 3 's local state in $s^{\prime}$, i.e., there is a state $s^{\prime \prime \prime \prime}$ such that $l_{3}\left(s^{\prime \prime \prime \prime}\right)=l_{3}\left(s^{\prime}\right)$ and

$l_{1}\left(s^{\prime \prime \prime \prime}\right)=\langle\langle(\mathbf{2}, 2,2),(\mathbf{1}, 6,5),(3,2,7),(4,4,12),(5,7,9)\rangle,\langle\rangle,\langle\rangle,\langle\rangle\rangle$

Now suppose that agent 1 discovers unit $m u_{3.1}$ 's position. The change in his local state is recorded in the global state $s^{\prime \prime}$ differing from $s^{\prime}$ as to $l_{1}$ :

$l_{1}\left(s^{\prime \prime}\right)=\langle\langle(1,2,2),(2,6,5),(3,2,7),(4,4,12),(5,7,9)\rangle,\langle\rangle,\langle(1,-3,-3)\rangle,\langle\rangle\rangle$

Then, agent 1 too knows that one of his units is within the range of enemy fire. In particular, she has de re knowledge of this fact, i.e., she knows the identity of agent 3's unit:

$\left(\mathcal{P}, s^{\prime \prime}\right) \models \exists_{1} z K_{1} \exists_{3} z^{\prime} F \operatorname{Dist}\left(z, z^{\prime}\right) \quad$ and also $\quad\left(\mathcal{P}, s^{\prime \prime}\right) \models \exists_{1} z \exists_{3} z^{\prime} K_{1} \operatorname{FDist}\left(z, z^{\prime}\right)$

Given that one of agent $i$ 's units is within the range of enemy fire, agent $i$ is in a dangerous situation. For expressing this, we introduce a predicate Danger, whose meaning is left underspecified through a caeteris paribus clause $\theta$. We assume that our QIS validates the following de dicto specification on Danger:

$\forall_{i} z\left(K_{i} \exists_{j} z^{\prime} F \operatorname{Dist}\left(z, z^{\prime}\right) \wedge \theta \leftrightarrow \operatorname{Danger}(z)\right)$, for $j \neq i$

The specification above is weaker than the following de re formula for specifying the Danger' predicate on danger:

$\forall_{i} z\left(\exists_{j} z^{\prime} K_{i} F \operatorname{Dist}\left(z, z^{\prime}\right) \wedge \theta \leftrightarrow \operatorname{Danger}^{\prime}(z)\right)$, for $j \neq i$

In fact, the notion of Danger' implies that of Danger, and agent 1 knows herself to be both in Danger and in Danger', while agent 3 only knows herself to be in Danger:

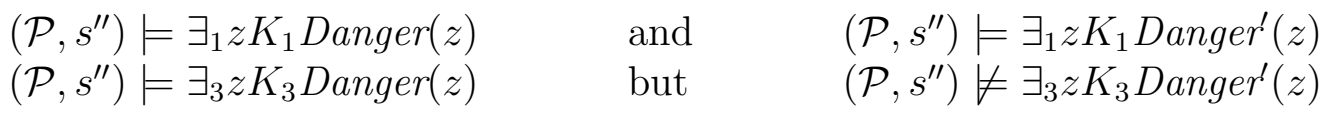

Agents can try to find a way out of this situation either by attacking or by withdrawing. In order to analyse these alternatives, we introduce a knowl- 
edge based protocol [17]. First of all, we define a predicate Access such that $\operatorname{Access}\left((x, y)\left(x^{\prime}, y^{\prime}\right)\right)$ iff $\left|x-x^{\prime}\right| \leq 1$ and $\left|y-y^{\prime}\right| \leq 1$. Intuitively, the set $\left\{p^{\prime} \mid \operatorname{Access}\left(p, p^{\prime}\right)\right\}$ contains the points recheable from $p$ in a single move. Moreover, we consider two actions: ATTACK and MOVE. The protocol for agent $i \neq j$ can be written in pseudo-code as follows:

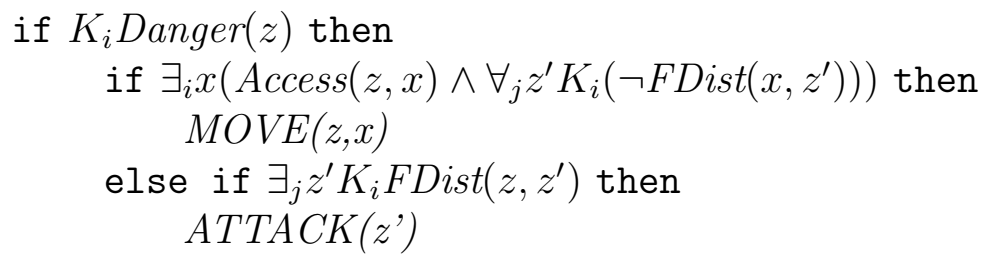

This protocol says that if agent $i$ knows that a unit $z$ is in danger, then she has to move it to an area known to be out of the enemy fire range. If this is not possible, then she has to attack the enemy unit threatening her own unit. Note that this protocol is extremely strict, as it requires knowledge of safety conditions before moving. In the present case, we have:

$$
\begin{aligned}
\left(\mathcal{P}, s^{\prime \prime}\right) \models \exists_{1} z\left(K_{1} \operatorname{Danger}(z)\right. & \wedge \forall_{1} x\left(\operatorname{Access}(z, x) \rightarrow K_{1} \exists_{3} z^{\prime} \operatorname{FDist}\left(x, z^{\prime}\right)\right) \wedge \\
& \left.\wedge \exists_{3} z^{\prime} K_{1} \operatorname{FDist}\left(z, z^{\prime}\right)\right)
\end{aligned}
$$

Therefore agent 1 , by following the knowledge-based protocol above, will attack the first unit of agent 3 .

By suitably extending our language, we could express interesting topological relationships on the various $D_{i}$, like the presence of obstacles. Moreover, we could introduce intensional predicates to describe in detail the characteristics of the military units. The system above is simply an example, but we deem it persuading enough to let us conclude that QIS are sufficiently expressive to represent a number of concrete MAS scenarios.

\subsection{Infinitely many muddy children}

In this subsection we use the language $\mathcal{L}_{n}^{o b j}$ to present an infinite version of the muddy children puzzle [17], a well-known scenario in epistemic logic. We observe that the solution to this puzzle depends only on the number $k$ of muddy children, not on the total number $m \geq k$ of children. In other words, at the end of the $k$-th round every child knows whether his forehead is muddy or not, independently from the total number $m$ of children.

This remark suggests that there is no need to fix in advance the size of the set $D$ of children in the muddy children puzzle. Given this, we take $D$ as containing an infinite number of children and we introduce an infinite version of this puzzle.

However, the language $\mathcal{L}_{n}^{o b j}$ in Section 3.1 contains only a finite number $n$ of epistemic operators, so in the present formalism we can reason about the 
knowledge of just a finite subset of $D$. This is a limitation of the present setting, which can be overcome by introducing a language with infinite denumerable epistemic operators. Such an extension is beyond our interest in this paper, so we leave it for further work.

We take the local state $l_{i}$ of child $i \leq n$ to be either 1 or 0 depending on whether his forehead is muddy or not, while the local state of the environment remains unspecified as it does not influence the present analysis. So we take it to be the empty sequence. We define a muddy children quantified interpreted system as follows:

Definition 58 A muddy children $Q I S$, or $M C Q I S$, is any pair $\langle S, D\rangle$, where $S \subseteq L_{e} \times L_{1} \times \ldots \times L_{n}$, and $D$ is an infinite set of children.

Note that we have a single quantification domain $D$ for all children, this implies that we can omit the subscript $i$ from quantifiers, and consider in $\mathcal{L}_{n}^{o b j}$ unique quantifiers $\forall, \exists$ for all agents.

Instead of using, as in the propositional case, propositions $p_{i}$ for $1 \leq i \leq n$, representing the fact that "child $i$ 's forehead is muddy", we use a unary predicative constant $M$ and infinitely many constants such that $M\left(a_{i}\right)$ intuitively means that "child $i$ 's forehead is muddy". Hereafter we present our analysis of this infinite version of the muddy children puzzle.

After the father's announcement that at least one child has a muddy forehead, the children have common knowledge of this fact. The language $\mathcal{L}_{n}^{o b j}$ has no common knowledge operator: we approximate this by assuming that $K_{i_{1}} \ldots K_{i_{j}} \exists x M(x)$ holds at step 1 , for every $i_{1}, \ldots, i_{j} \leq n$. This formula does not hold in every muddy children QIS, but it is valid on the MCQIS where $S$ does not contain the global state with only 0s.

Further, every child knows whether any other child is muddy or not, and this can be expressed in $\mathcal{L}_{n}^{o b j}$ as the following specification:

$$
\forall x\left(x \neq a_{j_{k}} \rightarrow\left(K_{j_{1}} \ldots K_{j_{k}} M(x) \vee K_{j_{1}} \ldots K_{j_{k}} \neg M(x)\right)\right)
$$

but he does not know whether he is muddy or not, that is, also the following specification holds at the beginning:

$$
\neg K_{j} M\left(a_{j}\right) \wedge \neg K_{j} \neg M\left(a_{j}\right)
$$

By induction on the number $k$ of muddy children, it is provable that at the $k$-th round of answers every child knows whether his forehead is muddy or not. Moreover, this result is independent from the actual number of children, and it holds also for an infinite number of children. In what follows we refer to principles and rules of inference of the system $Q . S 5_{n}^{o b j}$ in Section 4, we do not provide all the details of proofs, the interested reader should nonetheless be able to fill the gaps. 
For $k=1$, assume that child $j$ knows that (20) at least one child has a muddy forehead, and that (21) he cannot see any other child with a muddy forehead:

$$
\begin{array}{r}
K_{j} \exists x M(x) \\
K_{j} \forall y\left(y \neq a_{j} \rightarrow \neg M(y)\right)
\end{array}
$$

These formulas hold in the muddy children QIS where at least one and only one child has a muddy forehead. In the first-order modal system $Q . S 5_{n}^{o b j}$ we can show that child $j$ deduces that his own forehead is muddy as follows:

a. $K_{j}\left(\exists x M(x) \wedge \forall y\left(y \neq a_{j} \rightarrow \neg M(y)\right)\right)$ from (20), (21) by modal calculus

b. $K_{j} \exists x\left(M(x) \wedge\left(x \neq a_{j} \rightarrow \neg M(x)\right)\right) \quad$ from a by predicate calculus

c. $K_{j} \exists x\left(M(x) \wedge x=a_{j}\right) \quad$ from b by propositional calculus

So child $j$ acquires the knowledge that his own forehead is muddy, i.e., $K_{j} M\left(a_{j}\right)$ holds, and he will answer positively in the first round when asked.

For $k=2$, after the first round of negatively answered questions, every child $i$ knows that there are at least two children with muddy foreheads. We can represent this situation by means of the following de dicto formula:

$$
K_{i} \exists x, y(x \neq y \wedge M(x) \wedge M(y))
$$

Note that if child $i$ has not a muddy forehead, it is trivial to check that (22) holds, as child $i$ can see two other children with muddy foreheads. On the other hand, if child $i$ is one of the muddy children, then he can derive (22) in $Q . S 5_{n}^{o b j}$ from the principles (20), (21), (c) above.

In order to see this, assume that $j$ is the muddy child other than $i$, who has negatively answered the first question. This means that (c) $K_{j} M\left(a_{j}\right)$ fails, so either (20) or (21) does not hold. But it is the case that (20) $K_{j} \exists x M(x)$, so $\neg K_{j} \forall y\left(y \neq a_{j} \rightarrow \neg M(y)\right)$, that is, (21) is not valid.

Further, we can show that if child $j$ does not know that he is the only muddy child, then there is some other child, who is known to be muddy by $j$ :

$$
\neg K_{j} \forall y\left(y \neq a_{j} \rightarrow \neg M(y)\right) \rightarrow \exists y\left(y \neq a_{j} \wedge K_{j} M(y)\right)
$$

In fact, formula (23) can be proved in the system $Q . S 5_{n}^{o b j}$ as follows ${ }^{1}$ :
a. $\neg K_{j} \forall y\left(y \neq a_{j} \rightarrow \neg M(y)\right)$
by assumption
b. $\neg \forall y\left(y \neq a_{j} \rightarrow K_{j} \neg M(y)\right)$
from a by (see footnote)
c. $\exists y\left(y \neq a_{j} \wedge \neg K_{j} \neg M(y)\right)$
from by predicate calculus
d. $\exists y\left(y \neq a_{j} \wedge K_{j} M(y)\right)$
from c by (18)

\footnotetext{
$\overline{1}$ Note that $\left(t \neq t^{\prime} \rightarrow K_{j} \phi\right) \rightarrow K_{j}\left(t \neq t^{\prime} \rightarrow \phi\right)$ is a validity in all muddy children QIS, because of the interpretation of terms.
} 
As a result, (23) holds in the muddy children QIS. Therefore, by (23) and the negation of (21) we derive $\exists y\left(y \neq a_{j} \wedge K_{j} M(y)\right)$. Finally, by axioms $T$ and $N e c$ the muddy child $i$ acquires the knowledge that there is a muddy child different from $j$, i.e., $K_{i} \exists y\left(y \neq a_{j} \wedge M(y)\right)$. We also assumed that $i$ knows that $j$ has a muddy forehead, $K_{i} M\left(a_{j}\right)$, so we can easily derive (22) in $Q . S 5_{n}^{\text {obj }}$ by first-order calculus.

Up to now we showed that after the first round of questions every child, muddy or not, knows that there are at least two muddy children. Now, suppose that child $j$ can see only another child $z$ with a muddy forehead. This situation validates the following de re specification, i.e., child $j$ knows that every child other than him and $z$ is clean:

$$
\exists z K_{j} \forall k\left(k \neq a_{j} \wedge k \neq z \rightarrow \neg M(k)\right)
$$

We can show that from the de dicto knowledge of the existence of two muddy children expressed in (22), and formula (24) above, in $Q . S 5_{n}^{o b j}$ child $j$ deduces the de re knowledge that he is the other muddy child as follows:
a. $K_{j} \exists x, y \quad(x \neq y \wedge M(x) \wedge M(y)) \wedge$
$\wedge \exists z K_{j} \forall k \quad\left(k \neq a_{j} \wedge k \neq z \rightarrow \neg M(k)\right)$
from $(22),(24)$
b. $\exists z K_{j} \exists x, y(x \neq y \wedge M(x) \wedge M(y) \wedge$
$\left.\wedge \forall k\left(k \neq a_{j} \wedge k \neq z \rightarrow \neg M(k)\right)\right) \quad$ from a by predicate calculus
c. $\exists z K_{j} \exists x, y(x \neq y \wedge M(x) \wedge M(y) \wedge$
$\left.\wedge\left(x=a_{j} \vee x=z\right) \wedge\left(y=a_{j} \vee y=z\right)\right) \quad$ from b by $E x$
d. $\exists z K_{j} \exists x, y(x \neq y \wedge M(x) \wedge M(y) \wedge$
$\left.\wedge\left(\left(x=a_{j} \wedge y=z\right) \vee\left(y=a_{j} \wedge x=z\right)\right)\right)$ from c by distributivity
e. $\exists z K_{j} \quad\left(a_{j} \neq z \wedge M\left(a_{j}\right) \wedge M(z)\right)$
from d by predicate calculus

As a result, child $j$ acquires de re knowledge that he is the other muddy child and replies positively on the second round of questions.

It is an easy induction step to show that if there are $k$ muddy children, then there are formal proofs in the system $Q . S 5_{n}^{o b j}$ that they will know within the $k$-th round whether their foreheads are muddy. Since we are reasoning about an infinite set $D$ of children, none of the formulas above can be translated into the propositional language of [17] for example. Given the above, the infinite version of the muddy children puzzle has a natural and intelligible formal proof in first-order epistemic logic 


\section{Message-Passing QIS}

This section is devoted to modeling message-passing systems (MPS) [17,54] in the framework of objectual QIS. An MPS is a multi-agent system where agents communicate by exchanging messages, so the most relevant actions performed by agents are the sending and receiving of messages. The formalism of message passing systems is useful to model a wide range of multi-agent systems. For instance, a network of computers, such as the Internet, can be seen as an MPS. In general, any multi-agent system is an MPS if the message transmission delay is not negligible. In MPS the local state of each agent contains information about the initial state, the messages it has sent or received, as well as the internal actions it has taken. One of the first references dealing with MPS is [41], since then they have been extensively investigated both in their synchronous and asynchronous formulations $[17,54]$.

In what follows we show that MPS can be defined as a particular class of objectual SGS satisfying a finite number of specifications in the first-order modal language $\mathcal{L}_{n}^{D}$, where $n$ is the number of agents in the MPS. Our main result consists in showing that Proposition 4.4.3 in [17], concerning the knowledge of the ordering of events in MPS, can be restated as a validity on the class of QIS modeling MPS. Thus, the formalism of objectual QIS is powerful enough to deal with the theory of message passing systems. Throughout this section we refer to [17], par. 4.4.5-6, for details on MPS.

More formally, we introduce a set Act of actions $\alpha_{1}, \alpha_{2}, \ldots$, and a set $M s g$ of messages $\mu_{1}, \mu_{2}, \ldots$ For each agent $i \in A$, we consider a set $\Sigma_{i}$ of initial events $\operatorname{init}(i, \alpha)$, and a set $\operatorname{Int}_{i}$ of internal events $\operatorname{int}(i, \alpha)$. We define the local state $l_{i}$ for agent $i$ as a history over $\Sigma_{i}$, Int $t_{i}$ and $M s g$, that is, a sequence of events whose first element is in $\Sigma_{i}$, and whose following elements either belong to Int $_{i}$ or are events of the form $\operatorname{send}(i, j, \mu), \operatorname{rec}(i, j, \mu)$ for $j \in A, \mu \in M s g$. Intuitively, init $(i, \alpha)$ represents the event where agent $i$ performs the initial action $\alpha$, send $(i, j, \mu)$ represents the event where agent $i$ sends message $\mu$ to $j$, while the intended meaning of $\operatorname{rec}(i, j, \mu)$ is that agent $i$ receives message $\mu$ from $j$. Finally, $\operatorname{int}(i, \alpha)$ means that agent $i$ performs the internal action $\alpha$. A global state $s$ is a tuple $\left\langle l_{e}, l_{1}, \ldots, l_{n}\right\rangle$, where $l_{1}, \ldots, l_{n}$ are local states as above and $l_{e}$ contains all the events in $l_{1}, \ldots, l_{n}$.

We define a reflexive, transitive and anti-symmetric relation $\leq$ on the set of local states of any agent $i$ such that $l_{i} \leq l_{i}^{\prime}$ iff $l_{i}$ is a prefix of $l_{i}^{\prime}$. This order extends to global states, so that $s \leq s^{\prime}$ iff $l_{i} \leq l_{i}^{\prime}$ for every $i \in A$.

We define message passing QIS (MPQIS) as objectual quantified interpreted systems $\mathcal{P}=\langle S, D, I\rangle$ where $S$ is a non-empty set of global states, the domain $D$ of individuals includes all agents in $A$, the messages in $M s g$, the actions in Act, the events $e_{1}, e_{2}, \ldots$, and $I$ is an interpretation for language $\mathcal{L}_{n}^{D}$.

Intuitively, each MPQIS models the temporal evolution of a message passing system, which can be represented as a sequence $s_{0}, s_{1}, \ldots$ of global states such that $s_{0}=\left\langle\operatorname{init}\left(e, \alpha_{e}\right)\right.$, init $\left.\left(1, \alpha_{1}\right), \ldots, \operatorname{init}\left(n, \alpha_{n}\right)\right\rangle$, and for every $n \in \mathbb{N}$, 
either $s_{n+1}$ is identical to $s_{n}$ or there is $i \in A$ such that $l_{i}\left(s_{n}\right) \leq l_{i}\left(s_{n+1}\right)$ but $l_{i}\left(s_{n}\right) \neq l_{i}\left(s_{n+1}\right)$. Note that a single MPQIS can model several temporal evolutions of the same MPS.

We assume that our language has terms and predicative letters for representing the objects in the domain $D$ and the relations among them. In particular, $e_{1}, e_{2}, \ldots$ are metaterms ranging over events: for instance, we introduce $\forall e \phi[e]$ as a shorthand for

$$
\forall i, j, \mu, \alpha \phi[\operatorname{send}(i, j, \mu)] \wedge \phi[\operatorname{rec}(i, j, \mu)] \wedge \phi[\operatorname{init}(i, \alpha)] \wedge \phi[\operatorname{int}(i, \alpha)]
$$

In fact, every event is either a send event, a receive event, an initial action, or an internal action. We use the same notation for the objects in the model and the syntactic elements, the distinction will be made clear by the context. We observe that we can express event specifications by using formulas in $\mathcal{L}_{n}^{D}$, which are provably valid on every MPQIS (the existence of a unique individual $\exists$ ! can be defined by means of $=)$. For example, we can state:

$$
\begin{aligned}
& \forall e \exists ! i, j, \mu, \alpha \quad(i \neq j) \wedge(e=\operatorname{send}(i, j, \mu) \vee e=\operatorname{rec}(i, j, \mu) \vee e=\operatorname{init}(i, \alpha) \vee e=\operatorname{int}(i, \alpha)) \\
& \forall i, j, \mu, \alpha \exists ! e_{1}, e_{2}, e_{3}, e_{4}\left(\operatorname{send}(i, j, \mu)=e_{1} \wedge \operatorname{rec}(i, j, \mu)=e_{2} \wedge \operatorname{init}(i, \alpha)=e_{3} \wedge\right. \\
& \left.\quad \wedge \operatorname{int}(i, \alpha)=e_{4} \wedge e_{1} \neq e_{2} \wedge e_{1} \neq e_{3} \wedge e_{1} \neq e_{4} \wedge e_{2} \neq e_{3} \wedge e_{2} \neq e_{4} \wedge e_{3} \neq e_{4}\right)
\end{aligned}
$$

The first specification expresses the fact that every event is either a send event or a receive event, where the sender is different from the receiver, an initial action, or an internal action. Thus, it cannot be the case that $e=\operatorname{send}(i, j, \mu)=$ $\operatorname{send}\left(i^{\prime}, j^{\prime}, \mu^{\prime}\right)$ for distinct agents and messages. The second specification states that every send or receive event, initial action, and internal action are distinct events. Thus, we cannot have $\operatorname{send}(i, j, \mu)=e=\operatorname{rec}\left(i^{\prime}, j^{\prime}, \mu^{\prime}\right)$. It is easy to check that our definition of MPQIS validates both specifications.

In [17], p. 132 the authors list three key constraints on MPS, the third one involves the notion of run on a SGS, i.e., a temporal evolution of global states. Nonetheless, we can restate the first two without introducing runs:

MP1 a local state $l_{i}$ for agent $i$ is a history over $\Sigma_{i}, I n t_{i}$ and $M s g$;

MP2 for every event $\operatorname{rec}(i, j, \mu)$ in $l_{i}(s)$ there exists an event $\operatorname{send}(j, i, \mu)$ in $l_{j}(s)$.

Further, the following simplifying assumption is considered:

* all events in a given agent's local state are distinct, an agent can never perform the same action twice.

We show how to formalise these specifications in the present formalism. First, we introduce a predicate $H$ for happened such that $\left(\mathcal{P}^{\sigma}, s\right) \models H(e, i)$ iff $e$ is an event in the local state $l_{i}(s)$ of agent $i$. The formula $H(e)$ is a shorthand for $\exists i H(e, i)$. By definition of MPS we can show that $\left(\mathcal{P}^{\sigma}, s\right) \models H(e)$ iff $e$ is an event in $s$. Further, we define an order Prec on events as follows: 
$\left(\mathcal{P}^{\sigma}, s\right) \models \operatorname{Prec}\left(e, e^{\prime}, i\right) \quad$ iff $\quad\left(\mathcal{P}^{\sigma}, s\right) \models H(e, i) \wedge H\left(e^{\prime}, i\right)$ and

for all $s^{\prime} \leq s,\left(\mathcal{P}^{\sigma}, s^{\prime}\right) \models H\left(e^{\prime}, i\right) \rightarrow H(e, i)$.

The definition of $\operatorname{Prec}\left(e, e^{\prime}\right)$ can be obtained from the one above by disregarding the $i$ component:

$\left(\mathcal{P}^{\sigma}, s\right) \models \operatorname{Prec}\left(e, e^{\prime}\right) \quad$ iff $\quad\left(\mathcal{P}^{\sigma}, s\right) \models H(e) \wedge H\left(e^{\prime}\right)$ and

for all $s^{\prime} \leq s,\left(\mathcal{P}^{\sigma}, s^{\prime}\right) \models H\left(e^{\prime}\right) \rightarrow H(e)$.

We can insist on the events in a global state $s$ to be partially ordered by specifying that $\operatorname{Prec}\left(e, e^{\prime}\right)$ is a reflexive and transitive relation on the set of past events:

$$
\begin{array}{r}
H(e) \rightarrow \operatorname{Prec}(e, e) \\
\operatorname{Prec}\left(e, e^{\prime}\right) \wedge \operatorname{Prec}\left(e^{\prime}, e^{\prime \prime}\right) \rightarrow \operatorname{Prec}\left(e, e^{\prime \prime}\right)
\end{array}
$$

Moreover, $\operatorname{Prec}\left(e, e^{\prime}, i\right)$ can be defined as a linear order on the events in $l_{i}$, i.e., it is also anti-symmetric and total:

$$
\begin{array}{r}
\operatorname{Prec}\left(e, e^{\prime}, i\right) \wedge \operatorname{Prec}\left(e^{\prime}, e, i\right) \rightarrow\left(e=e^{\prime}\right) \\
H(e, i) \wedge H\left(e^{\prime}, i\right) \rightarrow \operatorname{Prec}\left(e, e^{\prime}, i\right) \vee \operatorname{Prec}\left(e^{\prime}, e, i\right)
\end{array}
$$

We define Linear $\left(\operatorname{Prec}\left(e, e^{\prime}, i\right)\right)$ as the conjunction of (25)-(28) above, expressing the fact that the relation $\operatorname{Prec}\left(e, e^{\prime}, i\right)$ is linear. Also, we define the first event as the minimal one with respect to $\operatorname{Prec}\left(e, e^{\prime}, i\right)$, that is,

$$
F s t(e, i)::=\forall e^{\prime}\left(H\left(e^{\prime}, i\right) \rightarrow \operatorname{Prec}\left(e, e^{\prime}, i\right)\right)
$$

Finally, the formulas $\operatorname{Sent}(i, j, \mu), \operatorname{Recd}(i, j, \mu), \operatorname{Init}(i, \alpha)$, and $\operatorname{Int}(i, \alpha)$ are shorthands for $H(\operatorname{send}(i, j, \mu)), H(\operatorname{rec}(i, j, \mu)), H(\operatorname{init}(i, \alpha))$, and $H(\operatorname{int}(i, \alpha))$ respectively. We can now formalise the specifications MP1-2 and ${ }^{*}$ as follows:

$$
\begin{aligned}
\mathrm{MP}^{*} & \operatorname{Linear}\left(\operatorname{Prec}\left(e, e^{\prime}, i\right)\right) \wedge \exists ! e(F \operatorname{st}(e, i) \wedge \exists \alpha(e=\operatorname{init}(i, \alpha))) \wedge \\
& \wedge \forall e(\neg F \operatorname{st}(e, i) \rightarrow \exists j, \alpha, \mu(e=\operatorname{int}(i, \alpha) \vee e=\operatorname{send}(i, j, \mu) \vee e=\operatorname{rec}(i, j, \mu))) \\
\text { MP2' } & \forall i, j, \mu(\operatorname{Recd}(i, j, \mu) \rightarrow \operatorname{Sent}(j, i, \mu))
\end{aligned}
$$

The validity of MP1* on MPQIS forces the local state of any agent $i$ to satisfy specifications MP1 and *; while by MP2' specification MP2 is satisfied.

Formulas MP1* and MP2' are the basic specifications for MPQIS, but we can consider further key constraints on message passing system. A property often required in the framework of MPS is channel reliability. Modified from [17], an MPS is reliable if every sent message is eventually received, or more formally:

MP4 if $\operatorname{send}(i, j, \mu)$ is in $l_{i}(s)$, then there exists a global state $s^{\prime}$ such that $\operatorname{rec}(j, i, \mu)$ is in $l_{j}\left(s^{\prime}\right)$. 
In the language $\mathcal{L}_{n}^{D}$ we can formalise this specification as follows:

$\mathrm{MP} 4 ' \forall j, \mu\left(\operatorname{Sent}(i, j, \mu) \rightarrow \neg K_{i} \neg \operatorname{Recd}(j, i, \mu)\right)$

In fact, if $\operatorname{send}(i, j, \mu)$ is in $l_{i}(s)$, by M4' $\left(\mathcal{P}^{\sigma}, s\right) \models \neg K_{i} \neg \operatorname{Recd}(j, i, \mu)$, this means that there exists a global state $s^{\prime}$ such that $\left(\mathcal{P}^{\sigma}, s^{\prime}\right) \models \operatorname{Recd}(j, i, \mu)$, that is, $\operatorname{rec}(j, i, \mu) \in l_{j}\left(s^{\prime}\right)$. Thus, MP4 holds. Note that MP4' is actually stronger than MP4 as the former requires that the local states of agent $i$ in $s$ and $s^{\prime}$ are identical.

Further, a relevant property of MPS concerns authentication: if agent $i$ receives a message $\mu$ from agent $j$, then $i$ knows that $\mu$ was actually sent by $j$. This specification can be expressed as

$$
\forall j, \mu\left(\operatorname{Recd}(i, j, \mu) \rightarrow K_{i} \operatorname{Sent}(j, i, \mu)\right)
$$

Finally, we may require that agents have perfect recall, i.e., to know everything that has happened to them:

$$
\forall e\left(H(e, i) \rightarrow K_{i} H(e, i)\right)
$$

It is easy to show that MPQIS satisfy authentication and perfect recall but not channel reliability. Of course, we could add this formula in any specification should channel reliability be required. We remark that all the specifications introduced so far are defined by means of only the predicative constants $H(e, i)$ and $\operatorname{Prec}\left(e, e^{\prime}, i\right)$.

We now prove the main result of this section: Proposition 4.4.3 in [17] can be restated as a validity on the class of MPQIS satisfying MP1, MP2, and *. We do not provide the full statement here, but we note that this metatheoretical result can be cast as a formula in the first-order epistemic language $\mathcal{L}_{n}^{D}$. First, we introduce a relation $\mapsto_{G}$ of potential causality between events, as discussed in $[41,54]$. This relation is intended to capture the intuition that event $e$ might have caused event $e^{\prime}$. Fix a non-empty subset $G$ of $A$, the relation $\mapsto_{G}$ holds between events $e, e^{\prime}$ at a state $s$ iff both $e$ and $e^{\prime}$ appear in $s$, and

(1) for some $i, j \in G, e^{\prime}$ is a receive event and $e$ is the corresponding send event;

(2) for some $i \in G$, events $e, e^{\prime}$ are both in $l_{i}(s)$, and either $e=e^{\prime}$, or $e$ comes earlier than $e^{\prime}$ in $l_{i}(s)$;

(3) for some event $e^{\prime \prime}$, we have that $e \mapsto_{G} e^{\prime \prime}$ and $e^{\prime \prime} \mapsto_{G} e^{\prime}$ hold at $s$.

Note that $\mapsto_{G}$ is a partial order on events. We say that $\left(\mathcal{P}^{\sigma}, s\right) \models e \mapsto_{G} e^{\prime}$ if $e \mapsto_{G} e^{\prime}$ hold at $s$ (we use the same notation for syntax and semantics).

Now we prove that the potential causality relation $\mapsto_{G}$ respects the order Prec of events by showing that the following validity holds in the class of MPQIS. This means that if event $e$ is the cause of event $e^{\prime}$, then it is distributed knowledge among the agents that $e$ happened before $e^{\prime}$. Note that this is the 
right to left implication of Proposition 4.4.3 in [17]:

Proposition $59 M P Q I S \models \forall e, e^{\prime}\left(\left(e \mapsto_{G} e^{\prime}\right) \rightarrow D_{G} \operatorname{Prec}\left(e, e^{\prime}\right)\right)$

Proof. Assume that $\left(\mathcal{P}^{\sigma}, s\right) \models e \mapsto_{G} e^{\prime}$. If $e^{\prime}$ is a receive event and $e$ is the corresponding send event, then $l_{i}(s)=l_{i}\left(s^{\prime}\right)$ for all $i \in G$ implies $\left(\mathcal{P}^{\sigma}, s^{\prime}\right) \models$ $H(e) \wedge H\left(e^{\prime}\right)$, and for $s^{\prime \prime} \leq s^{\prime},\left(\mathcal{P}^{\sigma}, s^{\prime \prime}\right) \models \operatorname{Recd}(i, j, \mu) \rightarrow \operatorname{Sent}(j, i, \mu)$ by MP2'. Thus, $\left(\mathcal{P}^{\sigma}, s\right) \models D_{G} \operatorname{Prec}\left(e, e^{\prime}\right)$.

If $e, e^{\prime}$ are both in $l_{i}(s)$ and either $e=e^{\prime}$ or $e$ comes earlier than $e^{\prime}$ in $l_{i}(s)$, then $l_{i}(s)=l_{i}\left(s^{\prime}\right)$ implies that $\left(\mathcal{P}^{\sigma}, s^{\prime}\right) \models H(e) \wedge H\left(e^{\prime}\right)$, and for $s^{\prime \prime} \leq s^{\prime}$, $\left(\mathcal{P}^{\sigma}, s^{\prime \prime}\right) \models H\left(e^{\prime}\right) \rightarrow H(e)$. It follows that $\left(\mathcal{P}^{\sigma}, s\right) \models K_{i} \operatorname{Prec}\left(e, e^{\prime}\right)$, and by axioms D1 and D2, $\left(\mathcal{P}^{\sigma}, s\right) \models D_{G} \operatorname{Prec}\left(e, e^{\prime}\right)$.

Finally, if there exists some event $e^{\prime \prime}$ such that $e \mapsto_{G} e^{\prime \prime}$ and $e^{\prime \prime} \mapsto_{G} e^{\prime}$, then we can assume without loss of generality that we are either in the first or second case above. In both cases $\left(\mathcal{P}^{\sigma}, s\right) \models D_{G} \operatorname{Prec}\left(e, e^{\prime \prime}\right) \wedge D_{G} \operatorname{Prec}\left(e^{\prime \prime}, e^{\prime}\right)$. Therefore, for every $s^{\prime}, l_{i}(s)=l_{i}\left(s^{\prime}\right)$ for all $i \in G$ implies $\left(\mathcal{P}^{\sigma}, s^{\prime}\right) \models H(e) \wedge H\left(e^{\prime}\right)$, and for $s^{\prime \prime} \leq s^{\prime},\left(\mathcal{P}^{\sigma}, s^{\prime \prime}\right) \models H\left(e^{\prime \prime}\right) \rightarrow H(e) \wedge H\left(e^{\prime}\right) \rightarrow H\left(e^{\prime \prime}\right)$. By transitivity, $\left(\mathcal{P}^{\sigma}, s^{\prime \prime}\right) \models H\left(e^{\prime}\right) \rightarrow H(e)$. Thus, $\left(\mathcal{P}^{\sigma}, s\right) \models D_{G} \operatorname{Prec}\left(e, e^{\prime}\right)$.

The analysis of message-passing systems carried out in this section shows the advantages in terms of expressivity of first-order epistemic formalisms in comparison with propositional ones. By means of language $\mathcal{L}_{n}^{D}$ we are able to formalise various constraints on MPS, thereby validating the suitability of the approach. Most importantly, an encoding of the right to left implication of Proposition 4.4.3 in [17] can be shown to be a validity on the class of QIS modeling MPS.

\section{Conclusions}

First-order epistemic logic allows for greater expressiveness in comparison to propositional formalisms. In specific applications this advantage can be critical for modeling agent systems in a more intuitive and effective way. Because of this, FOEL has successfully been applied to reasoning about knowledge in artificial intelligence $[42,53,45]$. However, the literature on this subject in the MAS area have so far fallen short of a deep and systematic analysis of the formal machinery (axiomatisability, decidability, completeness), even in the case of static epistemic logic.

In this paper we showed that the semantics of interpreted systems may naturally be extended to first-order, and we provided complete axiomatisations for several classes of structures, which is noteworthy given the known difficulties of these formalisms $[22,59]$. Specifically, we considered different assumptions on quantification domains, their relationship with the agents' local states, and the impact these assumptions make in terms of axiomatisations. We exempli- 
fied the use of these formalisms by investigating two examples. The puzzle of muddy children in Section 5 is part of the folklore of multi-agent systems in AI [17]; while the formalisation of the battlefield game as a quantified interpreted system is an original example of the expressive features of the present semantical framework. As regards message passing systems, the topic of Section 6, they have been extensively investigated in computer science both in their synchronous and asynchronous version. In [41] the relation $\mapsto_{G}$ between events is introduced, and in [54] it is used to defined a notion of common knowledge which is attainable also in asynchronous message passing systems. In Section 6 we followed [17] in the presentation of MPS, and proved that Proposition 4.4.3 can be cast as a validity on the class of MPQIS. This reassures us that the analysis is adequate.

We see this paper as a first contribution in the development of computationally grounded semantics for quantified epistemic logics. Indeed, extensions of the present framework seem worth pursuing. It may be of interest to explore the completeness issues resulting from term-indexing epistemic operators, as in [46]. This would greatly increase the expressive power of the formalism, so some limitation on index quantification might be introduced to retain a reasonable computational complexity. Another significant extension would be to add temporal operators to the language. This would open the way for axiomatisations of first-order temporal epistemic logics for MAS. However, any work in this area is likely to be affected by technical problems, especially with respect to axiomatisation and decidability $[28,64]$.

\subsection{Acknowledgements.}

The research described in this paper was partly supported by the EC Framework 6 funded project CONTRACT (IST Project Number 034418), by the reseach project "Logica Modale e Conoscenza" funded by the Scuola Normale Superiore, Pisa, and by the Royal Society through an International Joint Project award to both authors. The authors would like to thank the reviewers for useful comments on an earlier version of this paper. The first author would like to thank his PhD supervisor, Prof Massimo Mugnai, and Prof Giovanna Corsi, Prof Silvio Ghilardi, and Prof Achille Varzi for support and advice. The second author is grateful to Prof Marco Colombetti, Politecnico di Milano, for having introduced him to Quantified Modal Logic in 1994.

\section{References}

[1] F. Belardinelli. Quantified Modal Logic and the Ontology of Physical Objects, $\mathrm{PhD}$ thesis, Scuola Normale Superiore, Pisa, 2006. 
[2] F. Belardinelli, A. Lomuscio. A Quantified Epistemic Logic for Reasoning about Multi-Agent Systems, Proceedings of the 6th International Conference on Autonomous Agents and Multi-Agent systems (AAMAS07), pp. 589-591, IFAAMAS Press, 2007.

[3] F. Belardinelli, A. Lomuscio. Quantified Epistemic Logics with Flexible Terms, Proceedings of the Workshop on Logic, Rationality and Interaction (LORI), pp. 113-128, College Publications, 2007.

[4] F. Belardinelli, A. Lomuscio. A Complete Quantified Epistemic Logic for Reasoning about Message Passing Systems, Proceedings of the 8th International Workshop on Computational Logic in Multi-Agent Systems (CLIMA VIII), pp. 248-267, Springer Verlag, 2008.

[5] P. Bieber. A Logic of Communication in Hostile Environments, Proceedings of the 3rd IEEE Computer Security Foundations Workshop (CSFW'90), pp. 14-22, IEEE Computer Society, 1990.

[6] P. Blackburn, M. de Rijke, Y. Venema. Modal Logic, Cambridge University Press, 2001.

[7] P. Blackburn, J. van Benthem, F. Wolter (eds). Handbook of Modal Logic, Elsevier, 2006.

[8] A. Chagrov, M. Zakharyaschev. Modal Logic, Oxford University Press, 1997.

[9] E. Clarke, O. Grumberg, D. Peled. Model Checking, MIT Press, 1999.

[10] M. Cohen, M. Dams. A Complete Axiomatization of Knowledge and Cryptography, Logic in Computer science (LICS), pp. 77-88, IEEE Computer Society, 2007.

[11] P. Cohen, H. Levesque. Communicative Actions for Artificial Agents, Proceedings of the First International Conference on Multi-Agent Systems (ICMAS'95), pp. 65-72, AAAI Press, 1995.

[12] G. Corsi. A Unified Completeness Theorem for Quantified Modal Logics, Journal of Symbolic Logic, 67, pp. 1483-1510, 2002.

[13] G. Corsi. BF, CBF and Lewis Semantics, Logique et Analyse, pp. 103-122, 2005.

[14] A. Degtyarev, M. Fisher, B. Konev. Monodic Temporal Resolution, ACM Transactions on Computational Logic (TOCL), 7(1), pp. 108-150, 2006.

[15] A. Degtyarev, M. Fisher, A. Lisitsa. Equality and Monodic First-Order Temporal Logic, Studia Logica, 72, pp. 147-156, 2002.

[16] P. Dembiński, et al. Verics: A Tool for Verifying Timed Automata and Estelle Specifications, Proceedings of the 9th International Conference on Tools and Algorithms for the Construction and Analysis of Systems (TACAS03), pp. 278283, Springer Verlag, 2003. 
[17] R. Fagin, J. Halpern, Y. Moses, M. Vardi. Reasoning about Knowledge, MIT Press, 1995.

[18] R. Fagin, J. Halpern, M. Vardi. What can machines know? on the properties of knowledge in distributed systems, Journal of the ACM, 39(2), pp. 328-376, 1992.

[19] M. Fitting, R. Mendelsohn. First-order Modal Logic, Kluwer, Dordrecht, 1998.

[20] D. Follesdal. Knowledge, Identity, and Existence, Theoria, 23(1), pp. 1-27, 1967.

[21] M. Gammie, R. van der Meyden. MCK: Model Checking the Logic of Knowledge, Proceedings of the 16th International Conference on Computer Aided Verification (CAV04), pp. 479-483, Springer Verlag, 2004.

[22] J. Garson. Quantification in Modal Logic. In D. Gabbay and F. Guenthner (eds), Handbook of Philosophical Logic, 3, pp. 267-323, Reidel, 2001.

[23] J. Halpern, R. Fagin. Modelling Knowledge and Action in Distributed Systems, Distributed Computing, 3(4), pp. 159-179, 1989.

[24] J. Halpern, R. Shore. Reasoning about Common Knowledge with Infinitely Many Agents. Information and Computation, 191(1), pp. 1-40, 2004.

[25] Y. Hintikka. Knowledge, Identity, and Existence, Nous, 1(1), pp. 33-62, 1962.

[26] I. Hodkinson. Monodic Packed Fragment with Equality is Decidable, Studia Logica, 72, pp. 185-197, 2002.

[27] I. Hodkinson. Complexity of Monodic Guarded Fragments over Linear and Real Time, Annals of Pure and Applied Logic, 138, pp. 94-125, 2006.

[28] I. Hodkinson, F. Wolter, M. Zakharyaschev. Decidable Fragment of First-order Temporal Logics, Annals of Pure and Applied Logic, 106(1-3), pp. 85-134, 2000.

[29] I. Hodkinson, F. Wolter, M. Zakharyaschev. Decidable and Undecidable Fragments of First-order Branching Temporal Logics, Logic in Computer science(LICS), pp. 393-402, IEEE Computer Society, 2002.

[30] I. Hodkinson, R. Kontchakov, A. Kurucz, F. Wolter, M. Zakharyaschev. On the Computational Complexity of Decidable Fragments of First-order Linear Temporal Logics, Proceedings of the International Symposium on Temporal Representation and Reasoning (TIME), pp. 91-98, IEEE Press, 2003.

[31] W. van der Hoek, J. J. Ch. Meyer. Making Some Issues of Implicit Knowledge Explicit, International Journal of Foundations of Computer Science, 3(2), pp. 193-223, 1992.

[32] W. van der Hoek, J. J. Ch. Meyer, J. Treur. Formal Semantics of Temporal Epistemic Reflection Logic, Proceedings of the 4th International Workshops on Meta-Programming in Logic (META'94), pp. 332-352, Springer Verlag, 1994.

[33] G. Hughes, M. Cresswell. A New Introduction to Modal Logic, Routledge, 1996. 
[34] M. Huth, M. Ryan. Logic in Computer Science: Modelling and Reasoning about Systems, Cambridge University Press, 2004.

[35] M. Kaneko, T. Nagashima. Game Logic and its Applications 1, Studia Logica, 57, pp. 325-354, 1996.

[36] M. Kaneko, T. Nagashima. Game Logic and its Applications 2, Studia Logica, 58, pp. 273-303, 1997.

[37] S. Kripke. A Completeness Theorem in Modal Logic, Journal of Symbolic Logic, 24, pp. 1-14, 1959.

[38] T. Jager. An actualist semantics for quantified modal logic, Notre Dame Journal of Formal Logic, 23, pp. 335-349, 1982.

[39] G. Lakemeyer. Limited Reasoning in First-Order Knowledge Bases, Artificial Intelligence, 71(2), pp. 213-255, 1994.

[40] G. Lakemeyer. Limited Reasoning in First-Order Knowledge Bases with Full Introspection, Artificial Intelligence, 84(1-2), pp. 209-255, 1996.

[41] L. Lamport. Time, Clocks, and the Ordering of Events in a Distributed System, Communication of the ACM, 21(7), pp. 558-565, 1978.

[42] H. Levesque. Knowledge Representation and Reasoning, Annual Reviews Computer Science, 30(1), pp. 81-108, 1986.

[43] D. Lewis. On the Plurality of Worlds, Blackwell, 1986.

[44] Y. Liu, G. Lakemeyer, H. Levesque. A Logic of Limited Belief for Reasoning with Disjunctive Information, Proceedings of the 9th International Conference on Principles of Knowledge Representation and Reasoning (KR04), pp. 587-597, AAAI Press, 2004.

[45] Y. Liu, H. Levesque. Tractable Reasoning in First-order Knowledge Bases with Disjunctive Information, Proceedings of the 20th National Conference on Artificial Intelligence (AAAI-05), pp. 639-644, AAAI Press, 2005.

[46] A. Lomuscio, M. Colombetti. QLB: a Quantified Logic for Belief, Proceedings of the 3rd International Workshop on Agent Theories, Architectures, and Languages (ATAL-96), Springer Verlag, 1996.

[47] A. Lomuscio, M. Ryan. On the Relation between Interpreted Systems and Kripke Models. Proceedings of the AI97 Workshop on Theoretical and Practical Foundation of Intelligent Agents and Agent-Oriented Systems, Springer, 1997.

[48] Z. Manna, A. Pnueli. The Temporal Logic of Reactive and Concurrent Systems, vol. 1, Springer Verlag, 1992.

[49] K. McMillan. Symbolic Model Checking: An approach to the state explosion problem, Kluwer Academic Publishers, 1993.

[50] R. Meyden. Axioms for Knowledge and Time in Distributed Systems with Perfect Recall, Logic in Computer Science (LICS), pp. 448-457, IEEE Computer Society, 1994. 
[51] J.-J. Ch. Meyer, W. van der Hoek. Epistemic Logic for AI and Computer Science, Cambridge University Press, 1995.

[52] R. C. Moore. The Role of Logic in Knowledge Representation and Commonsense Reasoning, Proceedings of the 2nd National Conference of the American Association for Artificial Intelligence, pp. 428-433, AAAI Press, 1982.

[53] R. C. Moore. A Formal Theory of Knowledge and Action, in Formal Theories of the Commonsense World, Ablex Publishing Corp., 1984.

[54] P. Panangaden, K. Taylor. Concurrent Common Knowledge: defining agreement for asynchronous systems, Distributed Computing, 6(2), pp. 73-93, 1992.

[55] R. Parikh, R. Ramanujam. Distributed Processes and the Logic of Knowledge, Proceedings of the Conference on Logic of Programs, pp. 256-268, Springer Verlag, 1985.

[56] F. Raimondi, A. Lomuscio. Automatic Verification of Multi-agent Systems by Model Checking via OBDDs, Journal of Applied Logic, 5(2), pp. 235-251, 2007.

[57] A. Rao, M. Georgeff. Modeling Rational Agents within a BDI-Architectures, Proceedings of the 2nd International Conference on Principles of Knowledge Representation and Reasoning (KR'91), pp. 473-484, Morgan Kaufmann Publishers, 1991.

[58] M. Reynolds. Axiomatising First-order Temporal Logic: until and since over linear time, Studia Logica, 57(2/3), pp. 279-302, 1996.

[59] V. Shehtman. Completeness and Incompleteness in First-order Modal Logic: an Overview, Advances in Modal Logic, pp. 27-30, College Publications, 2006.

[60] H. Sturm, F. Wolter, M. Zakharyaschev. Monodic Epistemic Predicate Logic, Proceedings of the European Workshop on Logics in Artificial Intelligence, pp. 329-344, Springer Verlag, 2000.

[61] H. Sturm, F. Wolter, M. Zakharyaschev. Common Knowledge and Quantification, Economic Theory, 19, pp. 157-186, 2002.

[62] R. Thomason. Some Completeness Results for Modal Predicate Calculi, in Philosophical Problems in Logic, pp. 56-76. Reidel, 1980.

[63] F. Wolter. First Order Common Knowledge Logics, Studia Logica, 65, pp. 249$271,2000$.

[64] F. Wolter, M. Zakharyaschev. Decidable Fragments of First-order Modal Logics, Journal of Symbolic Logic, 66(3), pp. 1415-1438, 2001.

[65] F. Wolter, M. Zakharyaschev. Axiomatizing the Monodic Fragment of Firstorder Temporal Logic, Annals of Pure and Applied Logic 118(1-2), pp. 133-145, 2002 .

[66] M. Wooldridge. An introduction to multi-agent systems, John Wiley, 2002. 
[67] M. Wooldridge. Computationally Grounded Theories of Agency, Proceedings of the International Conference on Multi-Agent Systems (ICMAS), pp. 13-22, IEEE Press, 2000.

[68] M. Wooldridge. Reasoning about Rational Agents, MIT Press, 2000.

[69] M. Wooldridge. Verifying that Agents Implement a Communication Language, Proceedings of the 16th National Conference on Artificial Intelligence (AAAI99), pp. 52-57, AAAI Press, 1999.

[70] M. Wooldridge, M. Fisher, M. Huget, S. Parsons. Model Checking for Multiagent Systems: the Mable Language and its Applications. International Journal on Artificial Intelligence Tools, 15(2), pp. 195-226, 2006.

[71] M. Wooldridge, M. Fisher, M. Huget, S. Parsons. Model Checking Multiagent systems with MABLE; Proceedings of the First International Conference on Autonomous Agents and Multiagent Systems (AAMAS02), pp. 952-959, IFAAMAS, 2002.

[72] B. Wozna, A. Lomuscio. A Temporal Epistemic Logic with a Reset Operation. Proceeding of the 6th International Conference on Autonomous Agents and Multiagent Systems (AAMAS07), pp. 574-581, ACM, 2007. 